\title{
Structural Response Analysis of the Hydraulic Pneumatic Tensioner under its Local Failure Based on a Fully Coupled TLP-TTR System
}

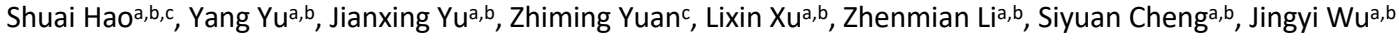 \\ a State Key Laboratory of Hydraulic Engineering Simulation and Safety, Tianjin University, Tianjin 300072, China \\ b Tianjin Key Laboratory of Port and Ocean Engineering, Tianjin University, Tianjin 300072, China \\ 'Department of Naval Architecture, Ocean \& Marine Engineering, University of Strathclyde, Glasgow G4 OLZ, UK
}

\begin{abstract}
:
The presented work studied the structural response of the hydraulic pneumatic tensioner (HPT) in a TLP-TTR system with failure of the tensioner cylinder. A fully coupled hull-tendon-TTR-tensioner model was established in AQWA to simulate the failure numerically. A specific HPT was modeled by considering 4 cylinders and the real-time stroke of each piston. A set of formulas was proposed to calculate the real-time tension including different components, e.g. Stribeck friction, in the tensioner. A riser array including 6 independent production TTRs and their tensioners were also modeled. The production TTR model was stacked up by different specific riser joints. The hydrodynamic force acting on the hull was obtained by using the 3D potential flow theory. The real-time tensions on different tensioner cylinders were obtained by using an in-house-developed program. Different environmental conditions, including a calm sea, regular waves, and extreme sea states, were considered in the simulations. In the results, the behaviors of different cylinders of the failed tensioner were presented. The results show that when an accidental local failure of the HPT occurs, the tension and stroke responses are still far from the designed-limits to induce a progressive failure.
\end{abstract}

\section{Keywords:}

Tensioner Failure; Top tension riser (TTR); Fully coupled model; Hydraulic pneumatic tensioner (HPT); Hydrodynamic response; 3D potential flow theory

\section{Introduction}

Tension Leg Platform (TLP) is a major type of offshore structures used in deep-water oil and gas field development. A TLP generally includes three parts: the hull (the main body of the platform floating on the sea); tendons (the crucial part to form tension legs, fastening the hull downward to wellhead on seabed. Generally, each leg consists of 2 or 3 tendons); and risers, top tension riser (TTR) in the case of TLP, used for oil drilling or production transfer. To maintain a sufficient top tension of the TTR in case of riser buckling due to the platform motions, there is a tensioner system between each TTR and the production deck of the hull to connect them together.

So far, there are two main types of tensioners applied in engineering: wireline riser tensioner (WRT) and direct-acting tensioner (DAT) (Lee et al., 2015). The hydraulic pneumatic tensioner (HPT) is a typical predominant DAT whose tension is directly acted on the tension ring of TTR by its hydraulic cylinder and piston (Wang and Liu, 2018). In general, there are 4 hydraulic cylinders in an HPT system. The tension in each pair of a cylinder and a piston is controlled independently by a corresponding set of hydraulic mechanism including high- and low-pressure accumulators (Chen et al., 2019), and the tensions in different cylinders are not influenced by each other. Therefore an HPT system of a TTR has 4 sets of independent hydraulic mechanisms. Most of the floating platforms have more than one riser and the number in some situation can reach more than a dozen. Consequently, dozens of the hydraulic mechanisms work on the platform simultaneously. Although failure of the hydraulic mechanism is an accidental event in the engineering field, the large total number of the hydraulic mechanisms available will increase its probability of occurrence, especially when the platform is experiencing extreme sea states. When a hydraulic mechanism fails, due to possible leakage or burst, the corresponding cylinder will lose partial or even full of its tension. Then the unbalanced total tension from the remaining cylinders leads to obviously different responses of the tensioner and riser compared with normal operational condition, which can cause structural damage to the platform. More extreme the sea state is, more serious the consequence would be. It is therefore necessary to study on the local failure of the HPT and to predict the subsequential structural responses.

The HPT and TTR are configured between the hull and seabed, thus the motion of the platform is their upper boundary condition. It is essential to use previous studies on hydrodynamic responses of floating platforms for reference. Over the last few decades, there are many scholars conducting study on that, especially on coupled hull-mooring-riser systems. Taylor and Jefferys (1986) performed a survey of diffraction/radiation analyses of a 
conventional TLP on behalf of the ISSC, which presented detailed frequency-domain hydrodynamic coefficients. It enabled researchers to validate a numerical model before launching a time-domain analysis. Lee and Newman (1991) evaluated the second-order loads based on extensions of the first-order panel method. Illustrative results were presented for the second-harmonic and sum-frequency diffraction loads on the ISSC TLP. Kim et al. (1994) presented results of a time-domain analysis of the nonlinear interaction and response of the coupled ISSC TLP system in the random seas. The first- and second-order wave exciting forces were calculated, and the nonlinear tendon dynamic analysis was also performed. The Morison equation was employed for the wave and current load on slender structures. However, their works focused more on the hull, while risers were not included to form a coupled platform-riser system. Lee et al. (2015) made a dynamic simulation of a wireline riser tensioner system of a mobile offshore drilling unit based on the multi-body system dynamics. Lee developed a WRT model on a semi-submersible platform, rather than a DAT, and its riser string is treated as a simplified elastic body. In fact, a riser is always composed of several joints of different functions and mechanical properties, which may influence the deformation of the riser in water. Wang and Liu (2018) performed numerical simulations in AQWA to investigate the dynamic response of a platform-riser coupled system with a hydro-pneumatic tensioner. A semi-submersible platform was connected to a TTR with a nonlinear tensioner. The overall coupling dynamic response under different wave conditions was investigated. But only one drilling TTR and a single-DOF (Degree of Freedom) tensioner were considered and modeled. The single-DOF tensioner model is a simplified model where the riser keeps vertical. A realistic HPT has 3 DOFs, which mean the riser can swing and be telescopic relatively to the platform and the HPT will provide axial and lateral restoring forces for the riser. Besides, for a production platform, there are commonly several risers configured under the main body. Therefore, the presented previous works are not sufficient for the presented study.

Some researchers have not coupled the riser with the floating platform, while concentrating on modeling the tensioner in detail. Chen et al. (2018b) generated a mathematical model of a hydro-pneumatic tensioner for TTR. The study considered the different parts of the hydraulic pressure system respectively, including cylinders, pistons, the high- and low-pressure accumulators, and the pressure loss in pipelines. It showed that the tension curve of the tensioner is still a nonlinear monotonic decreasing function of the piston stroke. Chen et al. (2019) discussed the key performance parameters of a hydro-pneumatic tensioner for the TTR. Based on the mathematical model of the tensioner, many further parameter sensitivity analyses were performed. Nevertheless, the model was still limited to the single-DOF tensioner. Chen et al. (2018a) proposed a modeling approach of the hydro-pneumatic tensioner for a TTR. Though 4 cylinders are considered as spring-damper elements, the platform motions at the top end of the TTR is provided by its global design, rather than putting the platform and the TTR into the whole coupled system. When modeling an HPT, the tension has a close relationship with the friction between the cylinder and the piston. With regard to the friction model, Andersson et al. (2007) developed some friction models for sliding dry, boundary, and mixed lubricated contacts. Among the models, there is a Stribeck model which is applicable to the lubricated sliding contacts. It combines static friction, Coulomb sliding friction, and viscous friction together. The total friction force is related to the direction and magnitude of the sliding speed. Marton and Lantos (2007) investigated on the modeling, identification, and compensation of stick-slip friction. Several empirical models were introduced to Stribeck friction. An experiment was designed to capture the Stribeck curves. Yanada and Sekikawa (2008) focused on the modeling of dynamic behaviors of friction. The friction behaviors were experimentally examined by using a hydraulic cylinder, especially under varying velocity conditions with the velocity reversal, which occurs all the time on riser tensioners. Tran et al. (2012) discussed the modeling of dynamic friction behaviors of a hydraulic cylinder. Friction characteristics were experimentally investigated under different external loads. A number of time-history curves were measured and presented.

However, few researchers focused on the riser tensioner failure. Although some design specifications for the tensioners require considering the structural redundancy, the works on predicting the failure response in the whole process has rarely been seen before, especially under specific environmental conditions. The lack of a fully coupled hull-tensioner-TTR model and the difficulty in modeling the fully coupled system contribute to the lack of interest regarding tensioner failure. Traditionally, simplified models (including linear spring and nonlinear spring, etc.) of riser tensioners are applied to other research. But they are not sufficient to solve the tensioner failure problem. A multi-cylinder tensioner model should be proposed and embedded to a fully coupled TLP-TTR system with the failed riser and other healthy risers (not the only one riser). Firstly, a multicylinder tensioner model can represent the failed cylinder and other intact cylinders of the same riser simultaneously; secondly, a multi-riser model can represent the riser with a local failure of its tensioner and other healthy risers; thirdly, the fully coupling effect between the platform hull and the TTRs is necessary to consider, because the riser tensioner is a complicated device connecting them together. Besides, more meticulous the fully coupled model is, including the specific configuration of the TTR, more accurate the response results will be.

In the presented study, a fully coupled TLP-TTR system is established in AQWA to simulate the local failure of an HPT numerically. A specific HPT is modeled by considering 4 cylinders and each piston stroke. A set of formulas to calculate the real-time tension including Stribeck friction in the tensioner is proposed. As for the TTR, there are three fundamental configurations: the drilling riser, the completion riser, and the production riser. Due to their different configurations and service durations, only the production riser is considered and simulated in the study. The production TTR model is stacked up by different riser joints including a tension joint, pup joints, a thick joint, a splash-zone joint, and a taper stress joint, etc. A casing pipe in the TTR is taken into account by an equivalent cross-section method. A TTR array including 6 independent risers and their tensioners is generated in the model. The hydrodynamic force acting on the hull of the TLP is obtained by using the 3D potential flow theory. Both of the force acted on the production deck of the hull by cylinders and the force acted on the tension ring of the TTR by pistons are obtained by using an in-housedeveloped code. The tensioner failure is also achieved by utilizing the in-house code. Different environmental conditions, including a calm sea, 
regular waves, and extreme sea states, are considered in simulations. In the results and discussions, the behaviors of different cylinders of the failed tensioner are presented. The tensioner responses in the healthy stage, transient stage, and steady stage are also developed and presented.

\section{TLP, Tendon, and TTR Configuration}

\subsection{Hull}

Currently, there are mainly 4 different forms of TLPs, including the conventional TLP, Seastar TLP, MOSES TLP, and the extended TLP. The target TLP selected in this study is an early-stage conventional TLP with 4 vertical cylindrical columns and 4 horizontal rectangular pontoons, as shown in Figure 1. It has the same superstructure as the one used by Taylor and Jefferys (1986).
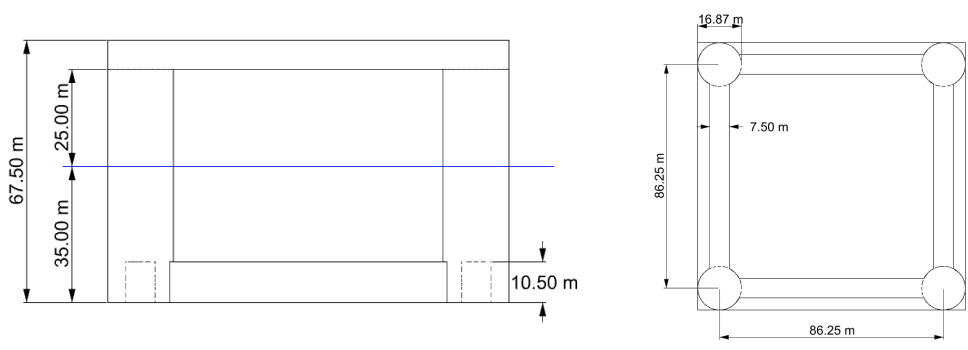

Figure 1 Hull dimensions

The main dimensions and configurations of the hull are summarized in Table 1.

Table 1 Hull configuration data

\begin{tabular}{|c|c|c|}
\hline HULL CONFIGURATION & UNIT & MAGNITUDE \\
\hline \multicolumn{3}{|l|}{ HULL PRINCIPAL DIMENSION } \\
\hline Spacing between column centers & $m$ & 86.25 \\
\hline Column radius & $\mathrm{m}$ & 8.44 \\
\hline Pontoon width & $\mathrm{m}$ & 7.50 \\
\hline Pontoon height & $\mathrm{m}$ & 10.50 \\
\hline Draft & $\mathrm{m}$ & 35.00 \\
\hline \multicolumn{3}{|l|}{ HULL INERTIA } \\
\hline Total mass & $\mathrm{t}$ & 40,500 \\
\hline Displacement & $\mathrm{t}$ & 54,500 \\
\hline Roll moment of inertia & $\mathrm{t} \cdot \mathrm{m}^{2}$ & $82.37 \mathrm{E}+06$ \\
\hline Pitch moment of inertia & $\mathrm{t} \cdot \mathrm{m}^{2}$ & $82.37 \mathrm{E}+06$ \\
\hline Yaw moment of inertia & $t \cdot m^{2}$ & $98.07 E+06$ \\
\hline Height of CoG above waterline & $\mathrm{m}$ & 3.00 \\
\hline
\end{tabular}

\subsection{Tendon}

The mooring system of the TLP includes 8 tendons, which are divided into 4 tension legs with 2 tendons each. The tendons are connected to the tendon porches which are installed at the bottom of the column, and the lower ends of the tendons are locked by subsea receptacles. In terms of

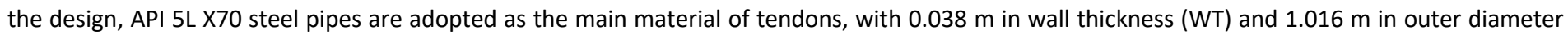
(OD). The pretension of each tendon is $1637.2 \mathrm{t}$.

\subsection{Top Tension Riser (TTR)}

A total of 12 TTRs are installed on the platform, which are arranged in an array of $3 \times 4$, as shown in Figure 2 . This study assumes that all the 12 TTRs are production TTRs. The stack-up of TTR joints and their cross section are shown in Figure 3. From the top to the bottom, the TTR consists of a production wellhead and a tree, a tension joint, an upper pup joint, a thick joint, a splash-zone joint, a lower pup joint, several standard joints, a taped stress joint with a tieback connector, and a subsea wellhead template with a guide post receptacle. A vertical tension joint is used to come across the production deck of the hull, and a tension ring is fixed at the tension joint to connect the pistons of the tensioner. The cross section of the TTR is a 2-layers casing pipe. The payload of each riser is $100.1 \mathrm{t}$. The top ends of the risers hang from the hydraulic tensioners. All of the subsea connection points of tendons and risers are all located on the seabed. 


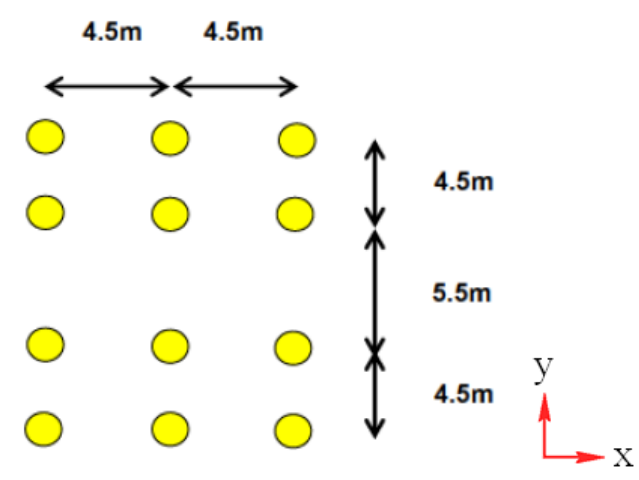

Figure 2 The layout of TTRs

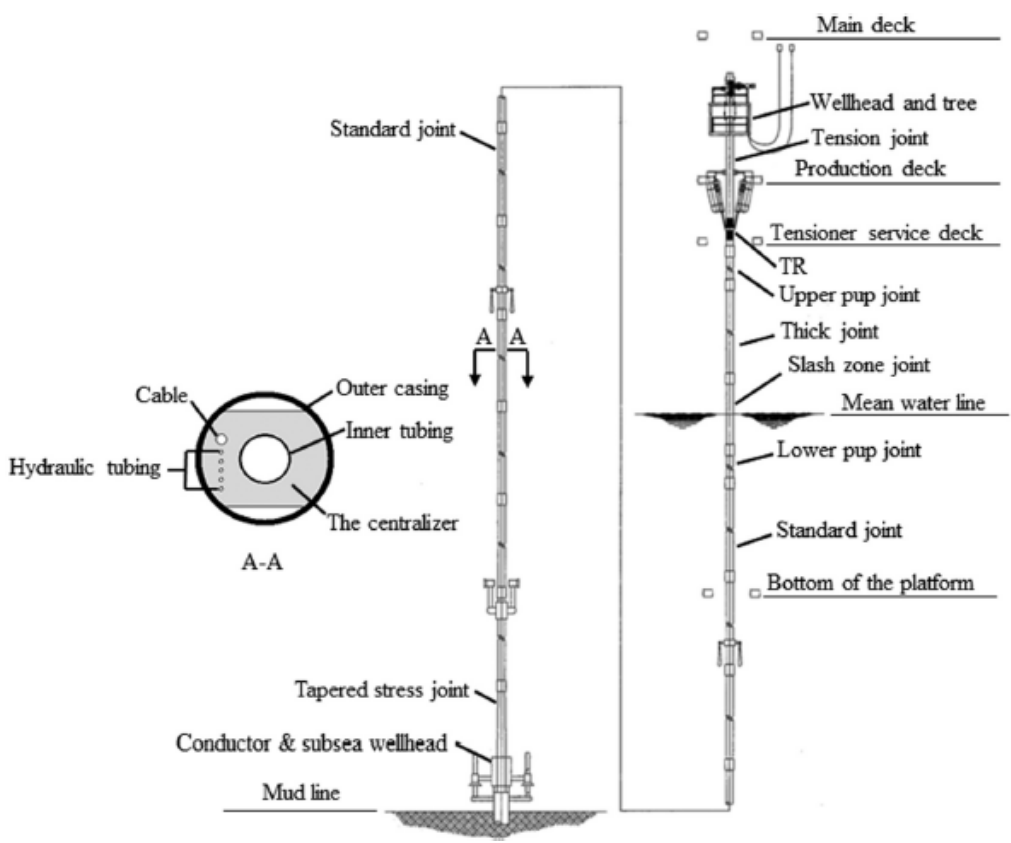

Figure 3 TTR joints and cross section sketch (Chen et al., 2018a)

\subsection{Hydraulic Pneumatic Tensioner}

For each TTR, a hydraulic pneumatic tensioner system is installed at the tension joint to connect the riser to the production deck. There are 4 pairs of hydraulic cylinders and pistons configured in each tensioner system. The front and vertical views of a tensioner are shown in Figure 4 . The cylinders hang from the cassette which is fixed on the production deck. The lower ends of the piston rods are connected with a tension ring from 4 diagonal directions. Each group of high- and low-pressure accumulators, a cylinder, a piston, and pipelines, comprises a hydraulic pressure mechanism to provide the tension based on the stroke for the corresponding cylinder independently. The production deck restrains the tension joint horizontally by 4 centralizer guide rollers. Generally, researchers assume that the guide rollers are fixed on the cassette. However, the TTR are free to move vertically and tilt around the guide rollers. 

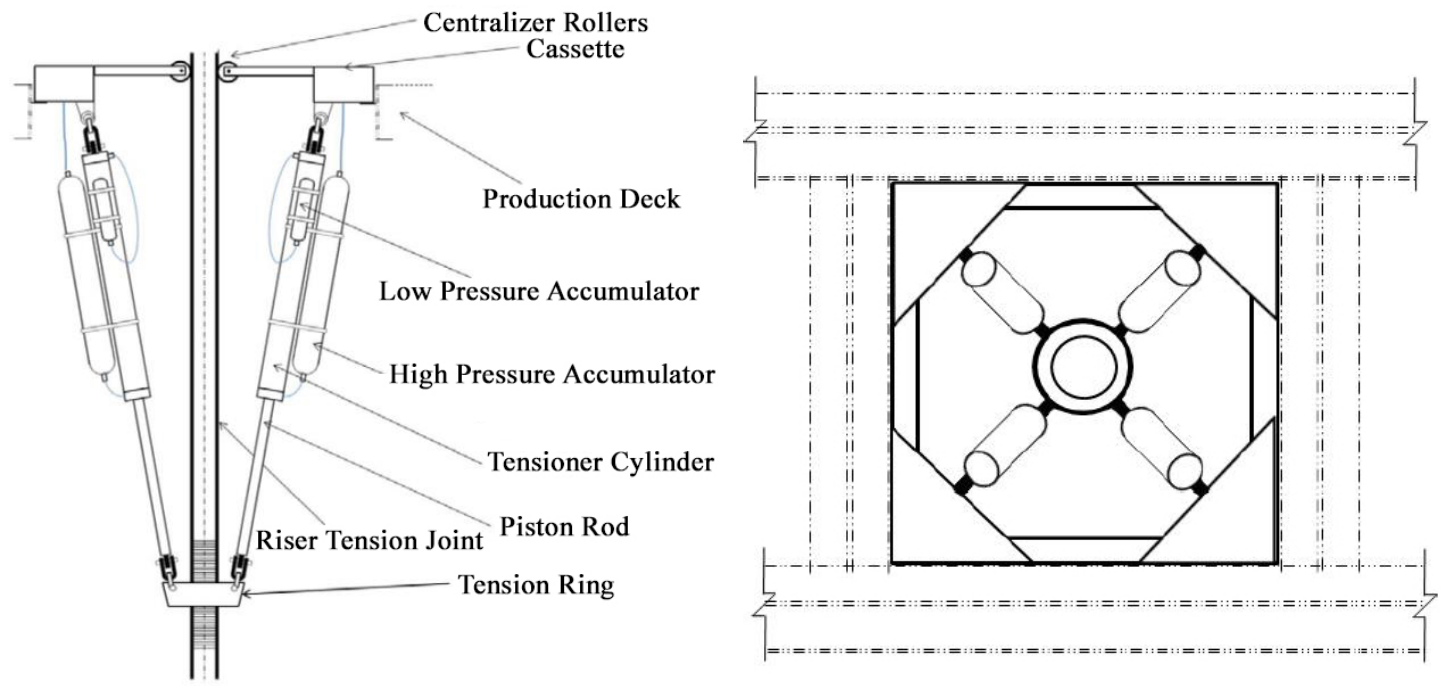

Figure 4 Hydraulic pneumatic tensioner front and vertical views

\section{Environmental Condition and Load Cases}

The operational water depth of the target platform is $450 \mathrm{~m}$. A calm sea, regular waves, and extreme sea states combined with irregular waves, winds, and currents are simulated to analyze the fully coupled system. The irregular waves are modeled by utiizing Jonswap spectrum. To obtain the behaviors of the tensioner in different conditions, these regular waves are of various periods, amplitudes, and directions. Details of the met-ocean are listed in Table 2 below. The TLP and the tensioners are symmetrical in both $x$ - and $y$-axes. Therefore, only the wave directions varying from $0^{\circ}$ and $45^{\circ}$ are considered. The wave load directions are defined in Figure 5, in XOY plane. In the presented study, all the sea load directions are defined as the direction to (rather than from) which the wave, wind or current moving forward.

Table 2 Details of the met-ocean

\begin{tabular}{|c|c|c|c|}
\hline Calm Sea & \multicolumn{3}{|c|}{-} \\
\hline \multirow{4}{*}{$\begin{array}{l}\text { Regular Wave } \\
\text { (Airy Wave) }\end{array}$} & Period (s) & Amplitude (m) & Direction \\
\hline & 10 & 8 & $0^{\circ}$ \\
\hline & 16 & 8 & $0^{\circ}$ \\
\hline & 10 & 8 & $45^{\circ}$ \\
\hline \multirow{18}{*}{$\begin{array}{l}\text { Extreme Sea State } \\
\text { (1000-yr Tropical Cyclone) }\end{array}$} & \multirow{5}{*}{ Irregular Wave } & Spectrum & Jonswap \\
\hline & & $\mathrm{Hs}(\mathrm{m})$ & 16.5 \\
\hline & & $\mathrm{Tp}(\mathrm{s})$ & 17.2 \\
\hline & & Direction & $0^{\circ} / 45^{\circ}$ \\
\hline & & Gamma & 2.4 \\
\hline & & Spectrum & NPD \\
\hline & & Elevation(m) & 10.0 \\
\hline & Wind & $\begin{array}{l}\text { Speed }(\mathrm{m} / \mathrm{s}) \text { in } 1 \\
\mathrm{hr} \text {. }\end{array}$ & 53.0 \\
\hline & & Direction & $0^{\circ} / 45^{\circ}$ \\
\hline & & Depth(m) & Velocity $(\mathrm{m} / \mathrm{s})$ \\
\hline & & 0 & 2.8 \\
\hline & & 23 & 2.63 \\
\hline & & 68 & 2.31 \\
\hline & Current & 113 & 1.99 \\
\hline & & 159 & 1.52 \\
\hline & & 204 & 1.37 \\
\hline & & 249 & 1.24 \\
\hline & & 294 & 1.14 \\
\hline
\end{tabular}




\begin{tabular}{|l|l|r|r|}
\hline \multirow{4}{*}{} & 340 & 1.06 \\
\cline { 3 - 4 } & & 385 & 1.04 \\
\cline { 3 - 4 } & & 450 & 0.73 \\
\cline { 3 - 4 } & Direction & $0^{\circ} / 45^{\circ}$ \\
\hline
\end{tabular}

\section{Numerical Model}

A composite model of the target TLP is constructed, which consists of a panel model, a Morison model, a tether model, and a 4-cylinder hydraulic pneumatic tensioner model, as shown in Figure 8. $T_{n}$ represents the $n$-th tendon; similar arrangement for $R_{n}$, and they are also shown in Figure 5. Finally, a fully coupled hull-tendon-TTR-tensioner model can be established for the hydrodynamic analysis under the tensioner failure (API, 2005, 2007; Faltinsen, 1993).

The motion equation of the floating structure in time domain can be written as below. In a regular wave, only the added mass and radiation damping, which are corresponding to a certain wave frequency, participate in the calculation:

$$
\left\{\boldsymbol{m}+\boldsymbol{A}\left(\omega_{0}\right)\right\} \ddot{\boldsymbol{X}}(t)+\boldsymbol{c}\left(\omega_{0}\right) \dot{\boldsymbol{X}}(t)+\boldsymbol{K} \boldsymbol{X}(t)=\boldsymbol{F}_{\text {hydrodynamic }}(t)+\boldsymbol{F}_{\text {tendon }}(t)+\boldsymbol{F}_{\text {tensioner }}(t)+\boldsymbol{F}_{\text {Morison }}(t)+\boldsymbol{F}_{\text {wind }}(t)(1)
$$

Where $\ddot{\boldsymbol{X}}, \dot{\boldsymbol{X}}, \boldsymbol{X}$ refer to the acceleration, velocity, and position of the structure respectively, $\boldsymbol{m}$ is the mass of the structure, $\boldsymbol{K}$ is the hydrostatic stiffness matrix, $\omega_{0}$ is the selected regular wave frequency. $\boldsymbol{A}(\omega)$ is the wave-frequency-based added mass, and $\boldsymbol{c}(\omega)$ is the wavefrequency-based radiation damping coefficient. Both of them are obtained by a standard frequency domain analysis. By comparison, in an irregular wave, all the frequency-based added mass and radiation damping should be considered in the time-based motion equation. It is realized by introducing a convolution integral term to calculate the time-based radiation force whose impulse response function is determined by all the added mass and radiation damping coefficients (Cummins, 1962):

$$
\left\{\boldsymbol{m}+\boldsymbol{A}_{\infty}\right\} \ddot{\boldsymbol{X}}(t)+\boldsymbol{K} \boldsymbol{X}(t)+\int_{0}^{t} \boldsymbol{R}(t-\tau) \ddot{\boldsymbol{X}}(\tau) d \tau=\boldsymbol{F}_{\text {hydrodynamic }}(t)+\boldsymbol{F}_{\text {tendon }}(t)+\boldsymbol{F}_{\text {tensioner }}(t)+\boldsymbol{F}_{\text {Morison }}(t)+\boldsymbol{F}_{\text {wind }}(t)(2)
$$

Where $\boldsymbol{A}_{\infty}$ is the added mass at infinite frequency, and it is an asymptotic value got from the above known frequency-based hydrodynamic coefficients. $\boldsymbol{R}$, as the acceleration impulse response function, is defined by:

$$
\boldsymbol{R}(t)=-\frac{2}{\pi} \int_{0}^{\infty} c(\omega) \frac{\sin (\omega t)}{\omega} d \omega=\frac{2}{\pi} \int_{0}^{\infty}\left\{A(\omega)-\boldsymbol{A}_{\infty}\right\} \cos (\omega t) d \omega
$$

The hydrodynamic force $\boldsymbol{F}_{\text {hydrodynamic }}$ includes the incident force, diffraction force of the 1st order wave force, and the steady drift force. For irregular waves, it also includes difference-frequency and sum-frequency force of the 2 nd order wave force. These different components can also be obtained by the frequency domain analysis. $\boldsymbol{F}_{\text {tendon }}$ refers to the mooring force acted on the hull by tendons. $\boldsymbol{F}_{\text {tensioner }}$ is the riser tensioner force acted on the production deck by cylinders and tension joints. $\boldsymbol{F}_{\text {Morison }}$ refers to the water viscosity force calculated by using the viscosity term of Morison equation, including the viscous damping and the viscous force of waves and currents. $\boldsymbol{F}_{\text {wind }}$ is the wind force calculated by applying wind force coefficient matrix. A sketch diagram for the analysis procedure in the study is illustrated in Figure 6 . In the time domain analysis, the hull and the TTR joints above its tension ring are regarded as two rigid bodies. The tendons and the TTR below the tension ring are modeled as flexible bodies. The TTR below the tension ring refers to the riser string between the tension ring and the seabed. A 4-cylinder tensioner model connects the rigid bodies together by interactive forces. A sketch of the fully coupled system is shown in Figure 7.

For solving the motion of rigid bodies, local structure axes (LSA) oxyz are introduced and fixed on the hull with the origin at its CoG, following with it to move. Another LSA $o^{\prime} x^{\prime} y^{\prime} z^{\prime}$ is fixed on the TTR above the tension ring, with the origin at its CoG. Fixed reference axes (FRA) $O X Y Z$ are placed at the origin of the model space which is on the calm sea surface, which is a global coordinate system and it never moves. The hull LSA and the global FRA are shown in Figure 5.

To solve the tensioner failure problem with 3D potential flow theory, an in-house developed subroutine based on FORTRAN and a Dynamic Link Library (DLL) is integrated into AQWA (Wang, 2007). The subroutine describes a real-time relationship between the tensioner forces and the tensioner motions by calling and return in every time step. 


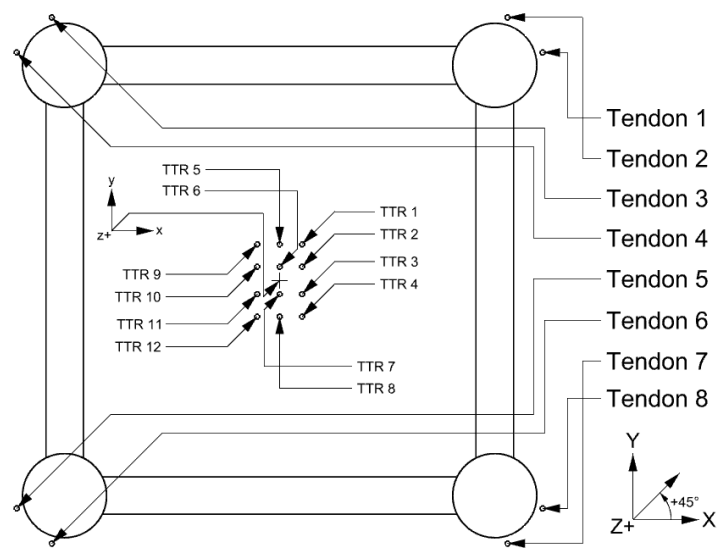

Figure 5 Tendon and TTR number, TLP heading, and sea load direction definition

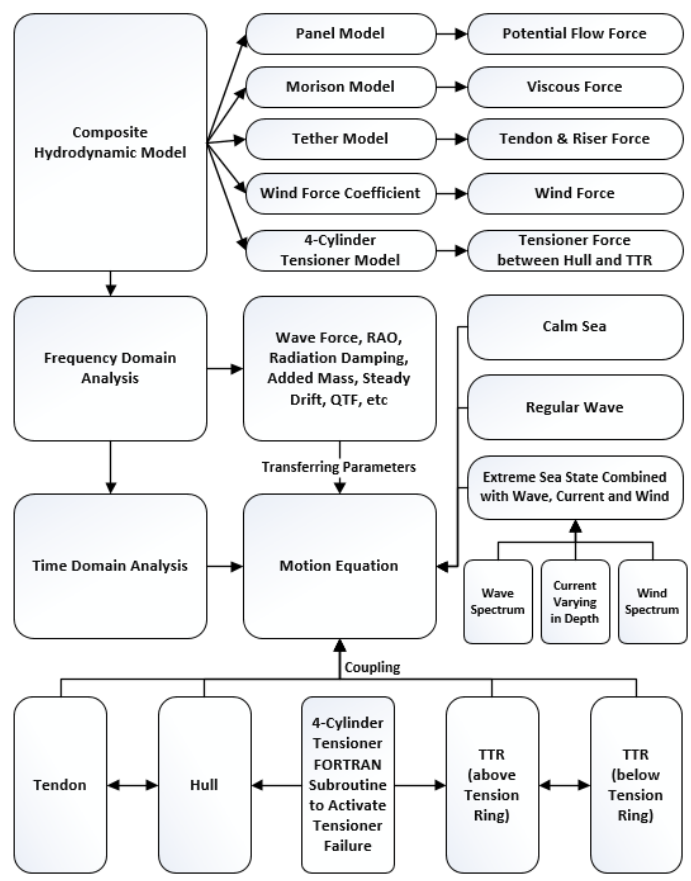

Figure 6 Sketch diagram for the analysis procedure 


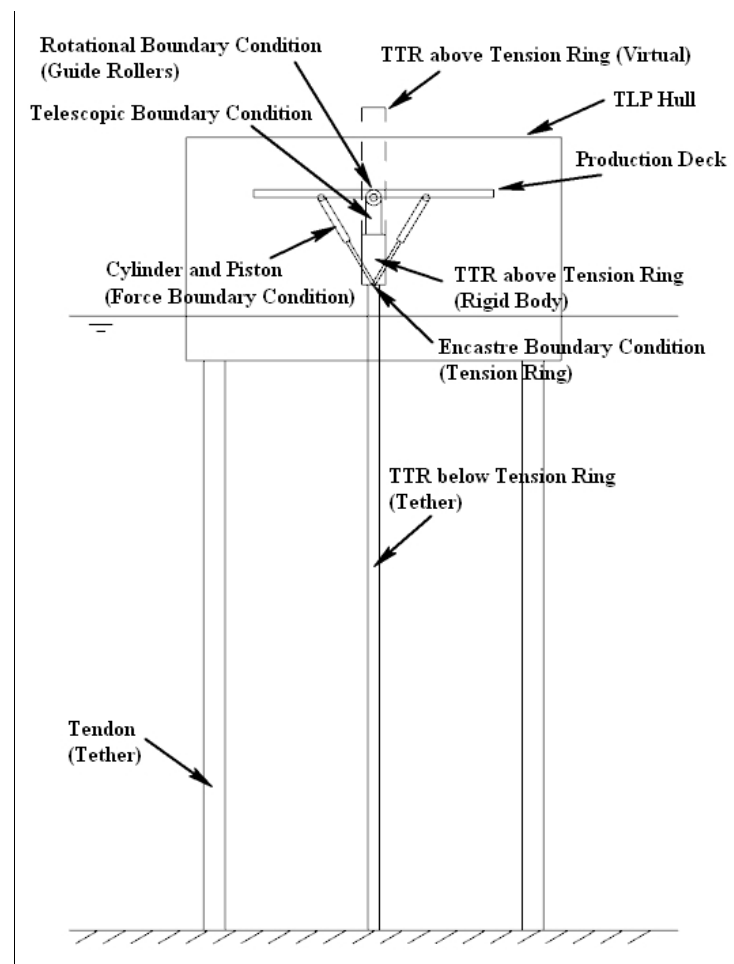

Figure 7 Sketch of the fully coupled system

\subsection{Panel Model}

A panel model describes the wet surface of the floating platform. The geometry of the hull is meshed into panels to calculate the hydrodynamic forces on it. The velocity potential due to the incident, diffraction, and radiation waves are written as:

$$
\varphi(\vec{X}) e^{-i \omega t}=\left[\left(\varphi_{1}+\varphi_{d}\right)+\sum_{j=1}^{6} \varphi_{r j} x_{j}\right] e^{-i \omega t}
$$

Eq (4) is used to get the hydrodynamic forces, including Froude-Krylov force due to the incident waves, the diffracting force due to the diffraction waves, and the radiation force due to a radiation wave induced by the motion of a rigid body of unit amplitude.

Besides, to calculate the second order wave excitation forces, the full difference- and sum-frequency quadratic transfer functions (QTFs) are used, which can be written as:

$$
\begin{aligned}
F_{s v}(t) & =\sum_{i=1}^{N S P L} \sum_{j=1}^{N S P L}\left\{P_{i j}^{-} \cos \left[-\left(\omega_{i}-\omega_{j}\right) t+\left(\varepsilon_{i}-\varepsilon_{j}\right)\right]+P_{i j}^{+} \cos \left[-\left(\omega_{i}+\omega_{j}\right) t+\left(\varepsilon_{i}+\varepsilon_{j}\right)\right]\right\} \\
& +\sum_{i=1}^{N S P L} \sum_{j=1}^{N S P L}\left\{Q_{i j}^{-} \sin \left[-\left(\omega_{i}-\omega_{j}\right) t+\left(\varepsilon_{i}-\varepsilon_{j}\right)\right]+Q_{i j}^{+} \sin \left[-\left(\omega_{i}+\omega_{j}\right) t+\left(\varepsilon_{i}+\varepsilon_{j}\right)\right]\right\}
\end{aligned}
$$

Where $P_{i j}$ and $Q_{i j}$ are the in-phase and out-of-phase components of the time independent transfer function, $\omega_{i}, \omega_{j}$ are the frequencies of each pair of wave components, $\varepsilon_{i}, \varepsilon_{j}$ are the random phase angles. The "-" or "+" signed in $P_{i j}$ and $Q_{i j}$ indicates either difference- or sum-frequency, respectively.

\subsection{Morison Model}

8 Morison members are added around the centerlines of the columns and pontoons, as the green solid lines indicated in Figure 8. Currently, it was a conventional way to consider the viscous force of water in a numerical simulation via the potential flow theory. The viscous force acted on the hull includes the viscous damping force due to its motion and the drag force due to waves and currents. Furthermore, Morison model is not only applied to the hull, but also to all the other slender bodies including the tendons and TTRs. Morison equation is expressed as below: 


$$
d F=\frac{1}{2} \rho D C_{d}\left|u_{f}-u_{s}\right|\left(u_{f}-u_{s}\right)+\rho A C_{m} \dot{u}_{f}-\rho A\left(C_{m}-1\right) \dot{u}_{s}
$$

Where $D$ is the characteristic drag diameter of the cross section, and $A$ is the area of the cross section. The drag coefficient $C_{d}$ and the inertia coefficient $C_{m}$ are set to be 1.1 and 2.0, respectively. $u_{f}$ is the transverse water particle velocity, and $u_{s}$ is the transverse structure velocity. $\dot{u}_{f}$ and $\dot{u}_{s}$ are their transverse accelerations. As the added mass is taken into account by using potential flow theory, the inertia terms are omitted when Morison equation is applied to the hull, while the full Morison equation is adopted for other slender bodies.
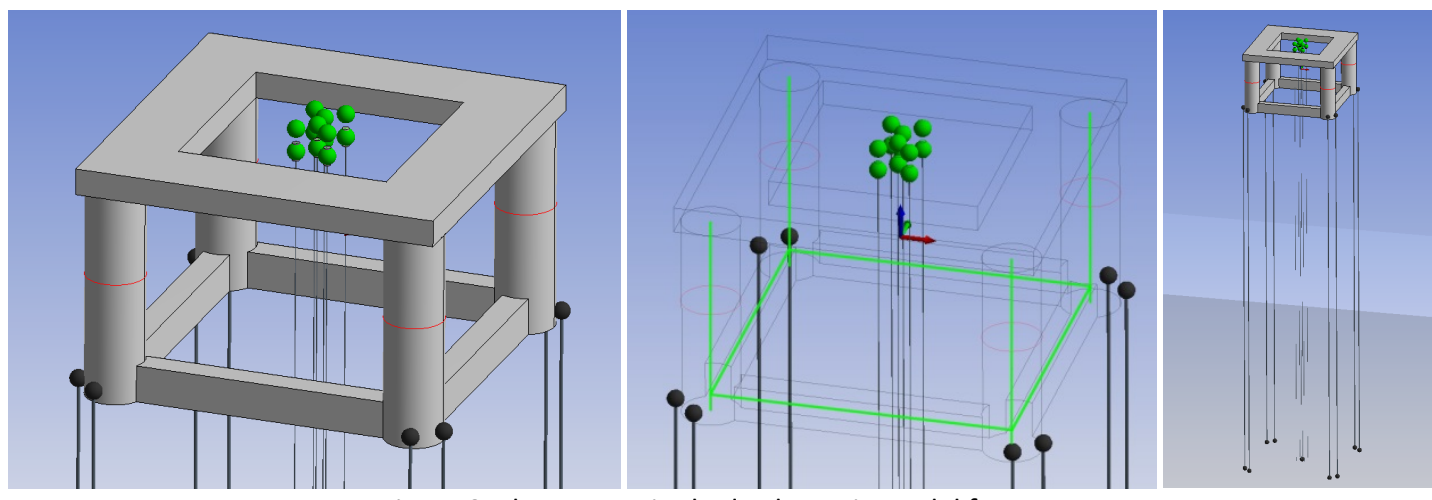

Figure 8 The composite hydrodynamic model for TLP

\subsection{Tether Model}

The tethers are modeled as flexible cylindrical tubes whose diameters are much smaller compared to the wavelength. The total force $\vec{F}_{e}$ applied on a tether element is expressed as:

$$
\vec{F}_{e}=\vec{F}_{k}+\vec{F}_{s}+\vec{F}_{i}+\vec{F}_{m}
$$

Where $\vec{F}_{k}$ is the internal force due to the structural bending stiffness, $\vec{F}_{s}$ represents the external forces applied by the springs at the end nodes, $\vec{F}_{i}$ represents the integrated forces, which consist of its gravity, hydrostatic forces, drag forces, wave inertia forces, and Froude-Krylov forces, and $\vec{F}_{m}$ is the force gained from calculation being performed in a moving reference frame. The tether element is substantially a beam element on which the bending moment and deflection are considered. The structural stiffness matrix is given to show the flexibility property of the tether elements, as below:

$$
\vec{K}=\frac{E I}{L^{3}}\left[\begin{array}{cccccccc}
12 & 0 & 0 & 6 L & -12 & 0 & 0 & 6 L \\
0 & 12 & -6 L & 0 & 0 & -12 & -6 L & 0 \\
0 & -6 L & 4 L^{2} & 0 & 0 & 6 L & 2 L^{2} & 0 \\
6 L & 0 & 0 & 4 L^{2} & -6 L & 0 & 0 & 2 L^{2} \\
-12 & 0 & 0 & -6 L & 12 & 0 & 0 & -6 L \\
0 & -12 & 6 L & 0 & 0 & 12 & 6 L & 0 \\
0 & -6 L & 2 L^{2} & 0 & 0 & 6 L & 4 L^{2} & 0 \\
6 L & 0 & 0 & 2 L^{2} & -6 L & 0 & 0 & 4 L^{2}
\end{array}\right]
$$

Where $L$ is the length of the tether element, $E$ is Young's modulus of elasticity, $I$ is the 2 nd area moment of the cross section. The nodal displacement matrix of the tether element can be expressed as:

$$
\vec{U}_{e}=\left[\begin{array}{llllllll}
u_{y, j} & u_{z, j} & \theta_{y, j} & \theta_{z, j} ; & u_{y, j+1} & u_{z, j+1} & \theta_{y, j+1} & \theta_{z, j+1}
\end{array}\right]^{T}
$$

So the term $\vec{F}_{k}$ in Eq. (7) is obtained from $\vec{F}_{k}=\vec{K} \vec{U}_{e}$. 
As mentioned in the beginning of Section 4, the tether model is adopted to describe the tendons and the lower parts of the TTRs, which should be regarded as flexible bodies. At the both ends of a tether, encastre conditions are set for tendons and the TTR. This boundary condition prevents any relative rotation of the tether ends around the connected structure, and the bending moment can be transferred. When a tether represents a TTR, its encastre top end is connected to the bottom of a rigid body which represents the TTR part above the tension ring. The study assumes that the TTR part above the tension ring is a rigid body that has a mass attribution, because it is much shorter than that below the tension ring. The local specific configuration of the tensioner is shown in Figure 4 and the global configuration of the TTR is shown in Figure 3 . The initial height of guide rollers and the tension ring are $30 \mathrm{~m}$ and $25 \mathrm{~m}$ above the waterline respectively. The TTR below the tension ring is modeled and stacked up by different specific riser joints. For the target TTR is a casing pipe, equivalent cross sections are selected for the tether models of these different joints, based on their same weight, same bending stiffness, and same hydrodynamic force, etc. The parameters of the detailed TTR model are listed in Table 3.

Table 3 Parameters of the detailed TTR model

\begin{tabular}{|c|c|c|c|c|c|}
\hline No. & Section Name & $\mathrm{OD}(\mathrm{m})$ & $\mathrm{WT}(\mathrm{m})$ & $L(m)$ & DW/WW $(\mathrm{kg} / \mathrm{m})$ \\
\hline- & Production Wellhead and Tree & \multirow{3}{*}{\multicolumn{4}{|c|}{$\begin{array}{c}\text { CoG: } 9.1 \mathrm{~m} \text { above Tension Ring } \\
\text { Weight: } 18.2 \mathrm{t}\end{array}$}} \\
\hline- & Tension Joint (Upper) & & & & \\
\hline- & Tension Ring & & & & \\
\hline 1 & Tension Joint (Lower) & 0.351 & 0.050 & 4.60 & 465.2 \\
\hline 2 & Upper Pup Joint & 0.279 & 0.014 & 2.70 & 142.4 \\
\hline 3 & Thick Joint & 0.279 & 0.014 & 9.20 & 142.4 \\
\hline 4 & Splash-zone Joint (Above) & 0.279 & 0.014 & 8.50 & 142.4 \\
\hline 5 & Splash-zone Joint (Below) & 0.279 & 0.014 & 4.30 & 79.6 \\
\hline 6 & Lower Pup Joint & 0.273 & 0.011 & 3.40 & 56.5 \\
\hline 7 & Standard Joint & 0.273 & 0.011 & 422.80 & 56.5 \\
\hline 8 & Taper Stress Joint (Top) & 0.273 & 0.011 & 5.00 & 42.7 \\
\hline 9 & Taper Stress Joint (Middle) & 0.313 & 0.031 & 5.00 & 216.3 \\
\hline 10 & Taper Stress Joint (Bottom) & 0.351 & 0.050 & 5.00 & 403.2 \\
\hline 11 & Subsea Wellhead & 0.762 & 0.051 & 4.50 & 690.4 \\
\hline
\end{tabular}

* The blue numbers in the last column indicate the wet weight (WW), while the black numbers indicate the dry weight (DW).

\subsection{Wind Force Coefficients}

Besides wave and current force, the wind feature of superstructure is considered and expressed by directional wind force coefficients. The matrix of wind force coefficients is listed in Table 4 as below. Among, X, Y, Z, RX, RY, and RZ refer to 6 DOFs respectively, i.e. surge, sway, heave, roll, pitch, and yaw.

Table 4 Wind force coefficients of TLP

\begin{tabular}{|c|c|c|c|c|c|c|}
\hline Dir & $x$ & $Y$ & Z & $\mathrm{RX}$ & RY & $\mathrm{RZ}$ \\
\hline$\left({ }^{\circ}\right)$ & \multicolumn{3}{|c|}{$\left(\mathrm{N} /(\mathrm{m} / \mathrm{s})^{2}\right)$} & \multicolumn{3}{|c|}{$\left(\mathrm{N} \cdot \mathrm{m} /(\mathrm{m} / \mathrm{s})^{2}\right)$} \\
\hline-180 & -4000 & 0 & 0 & 0 & -140000 & 0 \\
\hline-135 & -2970 & -2970 & 0 & 103950 & -103950 & 0 \\
\hline-90 & 0 & -4000 & 0 & 140000 & 0 & 0 \\
\hline-45 & 2970 & -2970 & 0 & 103950 & 103950 & 0 \\
\hline 0 & 4000 & 0 & 0 & 0 & 140000 & 0 \\
\hline 45 & 2970 & 2970 & 0 & -103950 & 103950 & 0 \\
\hline 90 & 0 & 4000 & 0 & -140000 & 0 & 0 \\
\hline 135 & -2970 & 2970 & 0 & -103950 & -103950 & 0 \\
\hline
\end{tabular}

\subsection{Hydraulic Cylinder Model}


A hydraulic cylinder model is applied to every pair of cylinder and piston. It provides the co-relations between the tension along the stroke and the relative motion, e.g. the stroke position and the stroke velocity. In general, the tension between a cylinder and a piston consists of 3 components, depending on the stroke position, the Stribeck friction, and the viscosity of lubricating oil, as following:

$$
T\left(x_{\text {stroke }}, v_{\text {stroke }}\right)=T_{\text {stroke }}\left(x_{\text {stroke }}\right)+T_{\text {strb }}\left(v_{\text {stroke }}\right)+T_{\text {visc }}\left(v_{\text {stroke }}\right)
$$

Where $x_{\text {stroke }}$ is the stroke position of a piston, $v_{\text {stroke }}$ is the stroke velocity, $T$ is the total tension on a single cylinder from the above mentioned 3 components, $T_{\text {stroke }}$ is the tension based on the stroke position, $T_{\text {strb }}$ is the Stribeck friction force, and $T_{\text {visc }}$ is the viscous friction force of the lubricating oil.

\section{Tension Based on Stroke}

As the largest component of the cylinder tension, the tension based on the stroke is a nonlinear curve versus the piston stroke, as the red line shown in Figure 15, which is fitted from the tensioner test data in its design information and the polynomial fitted formula is given in Eq (11). In the figure, the abscissa is on the top axis. The upper stroke limit is $0.610 \mathrm{~m}$, and lower stroke limit is $-2.134 \mathrm{~m}$, as the tensioner design data. When the piston is at the stroke of zero, the basic tension is $257 \mathrm{kN}$. The feature tallies with other research results even though the internal configuration of a hydropneumatic system is considered (Chen et al., 2018a; Chen et al., 2018b).

$$
\begin{aligned}
T_{\text {stroke }}\left(x_{\text {stroke }}\right)= & 257000-133143 \cdot x_{\text {stroke }}+53890 \cdot x_{\text {stroke }}^{2}-9619 \cdot x_{\text {stroke }}^{3}+10931 \cdot x_{\text {stroke }}^{4}(11) \\
& -18062 \cdot x_{\text {stroke }}^{5}-4437 \cdot x_{\text {stroke }}^{6}+11114 \cdot x_{\text {stroke }}^{7}+815 \cdot x_{\text {stroke }}^{8}-2147 \cdot x_{\text {stroke }}^{9}
\end{aligned}
$$

\section{Tension from Stribeck Friction}

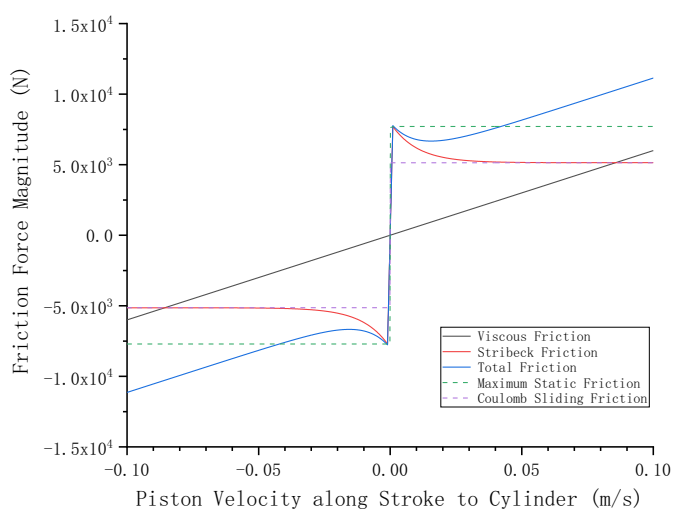

Figure 9 Different friction components

A Stribeck friction model is applied to consider the lubricated contact friction between a cylinder and a piston (Andersson et al., 2007):

$$
T_{\text {strb }}\left(v_{\text {stroke }}\right)=\left(F_{c}+\left(F_{s}-F_{c}\right) \cdot e^{-\left(\left|v_{\text {strokel }}\right| / v_{s}\right)}\right) \cdot \operatorname{sign}\left(v_{\text {stroke }}\right)
$$

Where $F_{c}$ is the Coulomb sliding friction force, and in terms of experience, $2 \%$ of the basic tension (the equilibrium tension) of the cylinder is chosen; $F_{s}$ is the maximum static friction force, and usually it is slightly larger than $F_{c}$, e.g. $3 \%$ of the basic tension; $v_{s}$ is the limit sliding velocity, and it is $0.01 \mathrm{~m} / \mathrm{s}$. The results of different friction components are illustrated in Figure 9.

\section{Tension from Viscosity}

Besides static and sliding frictions, the viscosity of lubrication cannot be neglected. The viscous friction force is expressed as following:

$$
T_{\text {visc }}\left(v_{\text {stroke }}\right)=k_{v} \cdot v_{\text {stroke }}
$$


Where $k_{v}$, viscous friction coefficient, is set to be $60 \mathrm{kN} /(\mathrm{m} / \mathrm{s})$ in the lubrication of the target tensioner.

\subsection{4-Cylinder Hydraulic Pneumatic Tensioner Model}

A 4-cylinder hydraulic pneumatic tensioner model is proposed on the fully coupled system. 4 pairs of cylinders and pistons are included in the tensioner model and the hydraulic cylinder model above is adopted for each pair. The real-time angles and the action points of these cylinders and pistons are taken into account. There is a total of 5 action points. 4 of them are located on the cassettes of the deck and only one is located the center axis of the TTR. The tensioner model is a force model expressed as a FORTRAN subroutine DLL. For the simulation, it is crucial to set a displacement boundary condition in AQWA that this rigid body is allowed to freely cross through the guide rollers fixed on the production deck, no matter what motions the platform and the TTR have. The complicated displacement boundary condition is designed and applied in the fully coupled model.

Besides an FRA, $O X Y Z$, and 2 LSAs, oxyz and $o^{\prime} x^{\prime} y^{\prime} z^{\prime}$, another LSA on the production deck with its origin at the equilibrium position of the tension ring, namely a tensioner LSA, is used to describe the tensioner behaviors, shown in Figure 10. The cylinder numbers and the projections in FRA of interactive forces are also shown. There are several basic steps to couple the TTR with the hull at each time step: 1) Obtain the motion parameters at the 5 action points; 2) Calculate the total tension force on each cylinder and then transfer them into $O X Y Z$; 3) Transfer the forces on $O X Y Z$ into $o x y z$ and $o^{\prime} x^{\prime} y^{\prime} z^{\prime}$; 4) Calculate the total external forces on the rigid bodies expressed in LSAs; 5) Transfer the total external forces back into the FRA and return to the main program.
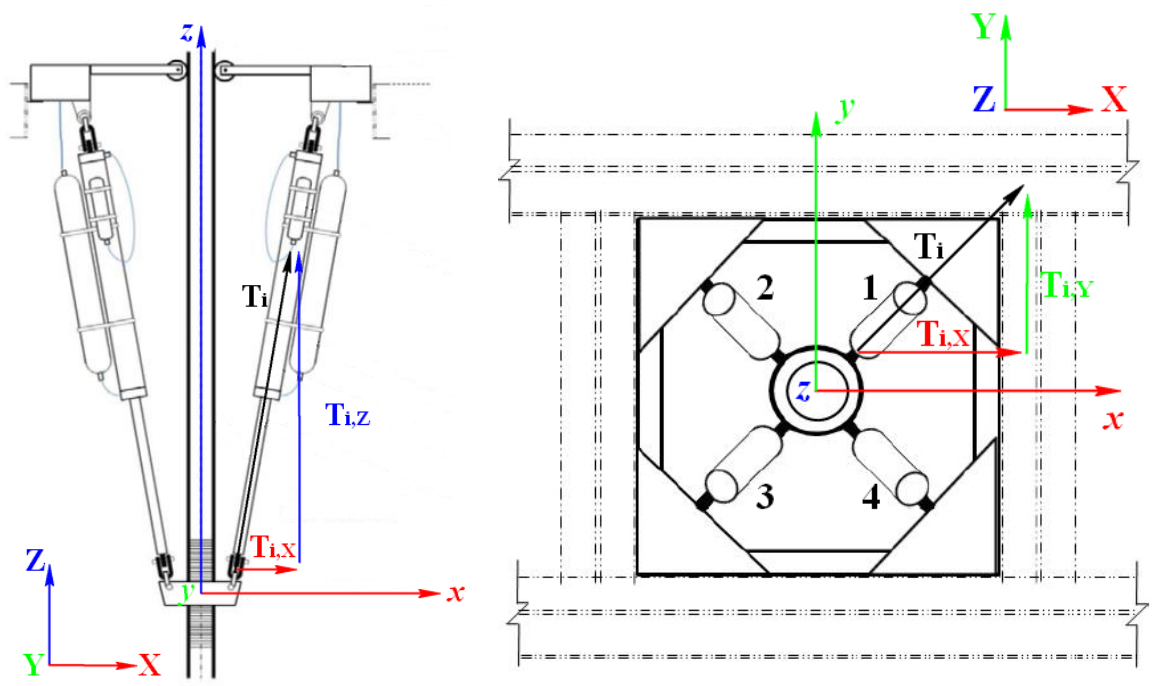

Figure 10 Front and top views of the Tensioner LSA

\section{Real-time Position of Tensioner}

1) The position of the hanging points of cylinders and guide rollers on the production deck is expressed as:

$$
\left[\begin{array}{c}
X_{i} \\
Y_{i} \\
Z_{i}
\end{array}\right]=\left[\begin{array}{c}
X_{g, \text { hull }} \\
Y_{g, \text { hull }} \\
Z_{g, \text { hull }}
\end{array}\right]+\boldsymbol{E}\left(\theta_{1, \text { hull }}, \theta_{2, \text { hull }}, \theta_{3, \text { hull }}\right)\left[\begin{array}{c}
x_{i}-x_{g, \text { hull }} \\
y_{i}-y_{g, \text { hull }} \\
z_{i}-z_{g, \text { hull }}
\end{array}\right] \quad(i=1, \ldots, 4, G R)(14)
$$

Where $i$ refers to the $i$-th cylinder; $G R$ stands for guide rollers; $\left[\begin{array}{ccc}X_{i} & Y_{i} & Z_{i}\end{array}\right]^{T}$ are the positions of the unknown points in FRA; $\left[\begin{array}{lll}X_{g, \text { hull }} & Y_{g, \text { hull }} & Z_{g, \text { hull }}\end{array}\right]^{T}$ is the CoG position of the hull in FRA; $\theta_{1, \text { hull }}, \theta_{2, \text { hull }}, \theta_{3, \text { hull }}$ is the Euler angles of the hull; $\left[\begin{array}{lll}x_{i} & y_{i} & z_{i}\end{array}\right]^{T}$ are the positions of the unknown points in LSA; $\left[\begin{array}{lll}x_{g, \text { hull }} & y_{g, \text { hull }} & z_{g, \text { hull }}\end{array}\right]^{T}$ is the CoG position of the hull in LSA; $\boldsymbol{E}$ is the Euler rotation matrix for an axis transformation.

2) The position of the tension ring is expressed as: 


$$
\left[\begin{array}{c}
X_{T R} \\
Y_{T R} \\
Z_{T R}
\end{array}\right]=\left[\begin{array}{c}
X_{g, p i s t} \\
Y_{g, p i s t} \\
Z_{g, p i s t}
\end{array}\right]+\boldsymbol{E}\left(\theta_{1, p i s t}, \theta_{2, p i s t}, \theta_{3, p i s t}\right)\left[\begin{array}{c}
x_{T R}-x_{g, p i s t} \\
y_{T R}-y_{g, p i s t} \\
z_{T R}-z_{g, p i s t}
\end{array}\right]
$$

Where $\left[\begin{array}{lll}X_{T R} & Y_{T R} & Z_{T R}\end{array}\right]^{T}$ is the unknown position; $\left[\begin{array}{lll}X_{g, p i s t} & Y_{g, p i s t} & Z_{g, p i s t}\end{array}\right]^{T}$ is the CoG position of the TTR the above the tension ring (TTR, hereafter, refers to the TTR rigid body in this section) in FRA; $\theta_{1, p i s t}, \theta_{2, p i s t}, \theta_{3, p i s t}$ are the Euler angles of the TTR; $\left[\begin{array}{lll}x_{T R} & y_{T R} & z_{T R}\end{array}\right]^{T}$ is the position of the tension ring in the LSA; $\left[\begin{array}{lll}x_{g, p i s t} & y_{g, p i s t} & z_{g, p i s t}\end{array}\right]^{T}$ is the CoG position of the TTR in the LSA.

3) The stroke of cylinders is expressed as:

$$
\begin{gathered}
L_{c y l_{i}}=\sqrt{\left(x_{T R}-x_{i}\right)^{2}+\left(y_{T R}-y_{i}\right)^{2}+\left(z_{T R}-z_{i}\right)^{2}} \quad(i=1, \ldots, 4) \\
x_{i, s \text { stroke }}=L_{c y l, 0}-L_{c y l_{i}} \quad(i=1, \ldots, 4)(17)
\end{gathered}
$$

Where $L_{c y l_{i}}$ is the total lengths of a cylinder and a piston from a hanging point to the tension ring, and $L_{c y l, 0}$ is this length when the tension ring is at the equilibrium position. $x_{i, \text { stroke }}$ is the stroke of the piston.

\section{Real-time Stroke Velocity of Tensioner}

The real-time stroke velocity scalar $v_{i, \text { stroke }}$ of a cylinder can be written as following. Firstly,

$$
\left[\begin{array}{c}
v_{i, X} \\
v_{i, Y} \\
v_{i, Z}
\end{array}\right]=\left[\begin{array}{c}
v_{\text {hull }, X} \\
v_{\text {hull }, Y} \\
v_{\text {hul }, Z}
\end{array}\right]+\left[\begin{array}{c}
v_{\text {hull }, R X} \\
v_{\text {hul }, R Y} \\
v_{\text {hull }, R Z}
\end{array}\right] \times\left[\begin{array}{c}
X_{i}-X_{g, \text { hull }} \\
Y_{i}-Y_{g, \text { hull }} \\
Z_{i}-Z_{g, \text { hull }}
\end{array}\right] \quad(i=1, \ldots, 4)
$$

Where $\left[\begin{array}{lll}v_{i, X} & v_{i, Y} & v_{i, Z}\end{array}\right]^{T}$ is the velocity vector of a hanging point in FRA; $\left[\begin{array}{llll}v_{\text {hull }, X} & v_{\text {hull }, Y} & v_{\text {hul }, Z}\end{array}\right]^{T}$ and $\left[\begin{array}{llll}v_{\text {hull }, R X} & v_{\text {hull }, R Y} & v_{\text {hul }, R Z}\end{array}\right]^{T}$ are the velocity vector at the CoG of the hull and the angular velocity of the hull in FRA respectively.

$$
\left[\begin{array}{c}
v_{T R, X} \\
v_{T R, Y} \\
v_{T R, Z}
\end{array}\right]=\left[\begin{array}{l}
v_{p i s t, X} \\
v_{p i s t, Y} \\
v_{p i s t, Z}
\end{array}\right]+\left[\begin{array}{l}
v_{p i s t, R X} \\
v_{p i s t, R Y} \\
v_{p i s t, R Z}
\end{array}\right] \times\left[\begin{array}{c}
X_{T R}-X_{g, p i s t} \\
Y_{T R}-Y_{g, p i s t} \\
Z_{T R}-Z_{g, p i s t}
\end{array}\right]
$$

Where $\left[\begin{array}{lll}v_{T R, X} & v_{T R, Y} & v_{T R, Z}\end{array}\right]^{T}$ is the velocity vector of the tension ring in FRA; $\left[\begin{array}{lll}v_{p i s t, X} & v_{p i s t, Y} & v_{p i s t, Z}\end{array}\right]^{T}$ and $\left[\begin{array}{lll}v_{p i s t, R X} & v_{p i s t, R Y} & v_{p i s t, R Z}\end{array}\right]^{T}$ are the velocity vector at the CoG of the TTR and the angular velocity of the TTR in FRA respectively. Then,

$$
v_{i, s t r o k e}=\frac{\left(\left[\begin{array}{c}
v_{i, X} \\
v_{i, Y} \\
v_{i, Z}
\end{array}\right]-\left[\begin{array}{c}
v_{T R, X} \\
v_{T R, Y} \\
v_{T R, Z}
\end{array}\right]\right) \cdot\left(\left[\begin{array}{c}
X_{i} \\
Y_{i} \\
Z_{i}
\end{array}\right]-\left[\begin{array}{c}
X_{T R} \\
Y_{T R} \\
Z_{T R}
\end{array}\right]\right)}{L_{c y l_{i}}} \quad(i=1, \ldots, 4)
$$

The stroke velocity is equal to the projected length of the relative velocity between a hanging point and the tension ring on the vector.

\section{Interactive Force on Hull}

Based on the single cylinder tension model expressed as Eq (10), the tension vector from each cylinder in FRA, $\boldsymbol{T}_{i, F R A}$, is written as: 


$$
\boldsymbol{T}_{i, F R A}=\left[\begin{array}{l}
\frac{T_{i}\left(x_{i, \text { stroke }}, v_{i, \text { stroke }}\right)}{L_{c y l_{i}}} \times\left(X_{T R}-X_{i}\right) \\
\frac{T_{i}\left(x_{i, \text { stroke }}, v_{i, \text { stroke }}\right)}{L_{c y l_{i}}} \times\left(Y_{T R}-Y_{i}\right) \\
\frac{T_{i}\left(x_{i, \text { stroke }}, v_{i, \text { stroke }}\right)}{L_{c y l_{i}}} \times\left(Z_{T R}-Z_{i}\right)
\end{array}\right] \quad(i=1, \ldots, 4)
$$

To transfer it from FRA into LSA, as below:

$$
\left[\begin{array}{c}
x \\
y \\
z
\end{array}\right]=\boldsymbol{E}^{T}\left(\theta_{1, \text { hull }}, \theta_{2, \text { hul }}, \theta_{3, \text { hull }}\right)\left[\begin{array}{l}
X \\
Y \\
Z
\end{array}\right](22)
$$

Where $\left[\begin{array}{lll}x & y & z\end{array}\right]^{T}$ is a vector expressed in LSA, $\left[\begin{array}{lll}X & Y & Z\end{array}\right]^{T}$ is the vector expressed in FRA. By moving the force vectors from their action points to the CoG of the hull, we will have:

$$
\boldsymbol{F}_{\text {hull }, \text { LSA }}=\sum_{i=1}^{4}\left[\begin{array}{c}
T_{i_{x}} \\
T_{i_{y}} \\
T_{i_{z}} \\
-\left(z_{i}-z_{g, \text { hull }}\right) T_{i_{y}}+\left(y_{i}-y_{g, \text { hull }}\right) T_{i_{z}} \\
\left(z_{i}-z_{g, \text { hull }}\right) T_{i_{x}}-\left(x_{i}-x_{g, \text { hull }}\right) T_{i_{z}} \\
-\left(y_{i}-y_{g, \text { hull }}\right) T_{i_{x}}+\left(x_{i}-x_{g, \text { hull }}\right) T_{i_{y}}
\end{array}\right]
$$

Where $\boldsymbol{F}_{\text {hul }, L S A}$ represents a force vector and a moment vector in the LSA of the hull. To transfer it back from LSA into FRA, the following expression can be used:

$$
\left[\begin{array}{l}
X \\
Y \\
Z
\end{array}\right]=\boldsymbol{E}\left(\theta_{1, \text { hull }}, \theta_{2, \text { hull }}, \theta_{3, \text { hull }}\right)\left[\begin{array}{c}
x \\
y \\
z
\end{array}\right]
$$

\section{Interactive Force on TTR}

The summed tension vector acting on the tension ring in FRA, $\boldsymbol{T}_{T R, F R A}$, can be expressed as:

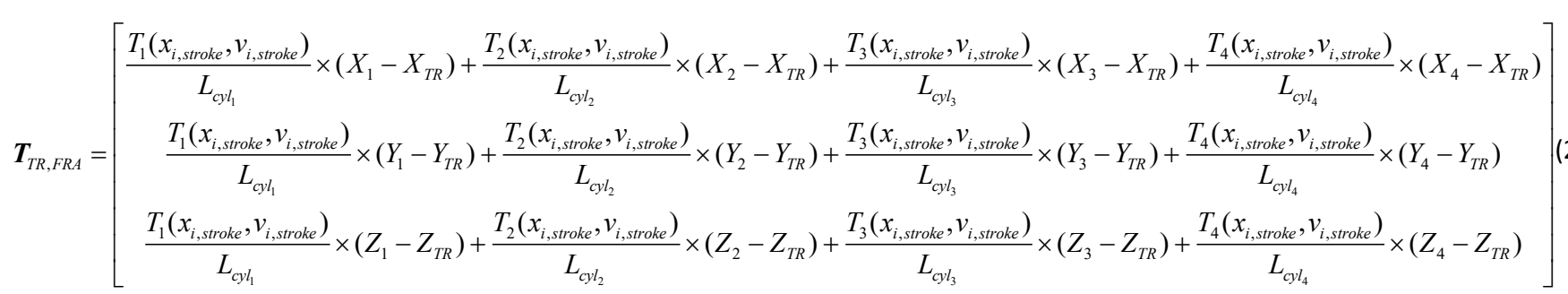

Similar to the interactive force on the hull, it can be transferred from the FRA into the TTR LSA. By moving the force vector from its action point to the CoG of the TTR, we will get: 


$$
\boldsymbol{F}_{T R, L S A}=\left[\begin{array}{c}
T_{T R_{x}} \\
T_{T R_{y}} \\
T_{T R_{z}} \\
-\left(z_{T R}-z_{g, p i s t}\right) T_{T R_{y}}+\left(y_{T R}-y_{g, p i s t}\right) T_{T R_{z}} \\
\left(z_{T R}-z_{g, p i s t}\right) T_{T R_{x}}-\left(x_{T R}-x_{g, p i s t}\right) T_{T R_{z}} \\
-\left(y_{T R}-y_{g, p i s t}\right) T_{T R_{x}}+\left(x_{T R}-x_{g, p i s t}\right) T_{T R_{y}}
\end{array}\right]
$$

Where $\boldsymbol{F}_{T R, L S A}$ is in the LSA of the TTR. It can be transferred back from the LSA into the FRA. Combining the 4 parts of above equations, the mathematical model of the tensioner can be universally applied to any motion of the hull and the tension ring, as long as they satisfy the displacement boundary condition above mentioned, without a simplification of the motion or constraint in any DOFs. It is the advantages of the fully coupled system.

\section{TTR Array}

The target TLP has a TTR array with 12 risers, while AQWA has a limitation of the maximum number of rigid bodies. Finally, to consider the riser with a tensioner failure and other healthy risers in the TTR array simultaneously, 6 independent TTRs and their HPTs are modeled separately. The details of the 6 risers and their tensioners are shown in Figure 11 . There are 4 risers, i.e. $R_{1}, R_{4}, R_{9}$, and $R_{12}$, at the corners and 2 risers, i.e. $R_{6}$ and $R_{7}$, at the center of the array. They are placed at the different horizontal positions in the TTR array to demonstrate their slightly different responses, even if all the risers are healthy.
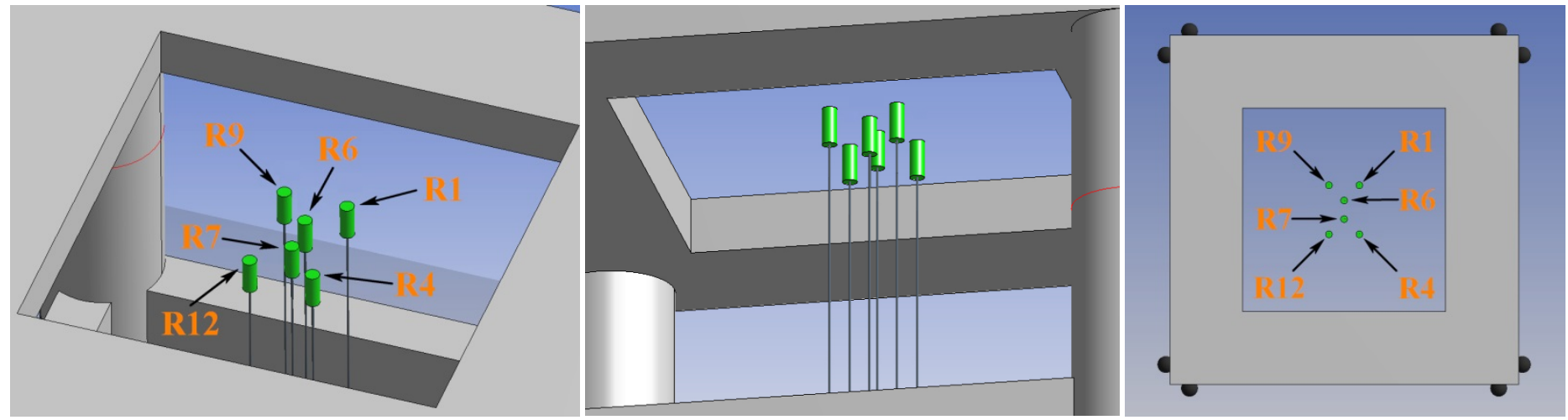

Figure 11 Riser array including 6 TTRs

\subsection{Local Tensioner Failure}

The local failure of the HPT hydraulic system was obtained by setting the cylinder tension to zero at the time of failure. Because only the hydraulic pressure mechanism fails, e.g. the high-pressure accumulator with its pipeline leaks or bursts, the cylinder loses the tension based on its stroke, while the tensions from the Stribeck friction and the viscosity are still retained. Under all the load conditions in the study, Cylinder 1 of $R_{1}$ is selected to fail at the 500-th second from the beginning of the numerical simulation. It should be noted that making the tension based on the stroke be zero is to analyze the failure response of the HPT on a more dangerous side. Losing all the tension of the cylinder in real life is an extreme situation, more commonly, when an accidental leakage appears on the hydraulic pressure mechanism, it usually loses a part of the tension.

\section{Hydrodynamic Model Validation}

An absolute calm sea condition is applied in some tests, e.g. static offset test, free decay test, etc. The main purpose to perform the static offset test and the free decay test is to verify the hydrostatic, hydrodynamic features, and the tendon mooring system of the TLP model. Moreover, all the hydrodynamic force and coefficients (e.g., wave excitation forces, motion responses, radiation damping, and added masses, etc.) of the hull model in frequency domain are validated by a reference paper (Taylor and Jefferys, 1986). Besides, a tensioner test for a set of 4 combined cylinders is performed to obtain its tension curves.

\subsection{Static Offset Test}

The restoring force versus the offset and set-down of the TLP hydrodynamic model is tested in a $45^{\circ}$ direction, shown in Figure 12 . The tension curves of the corresponding tendon $5 / 6$ and $1 / 2$ are also presented in Figure 13. The horizontal restoring force is in the opposite direction with the displacement, which consists of $F_{X}$ and $F_{Y}$ components. 


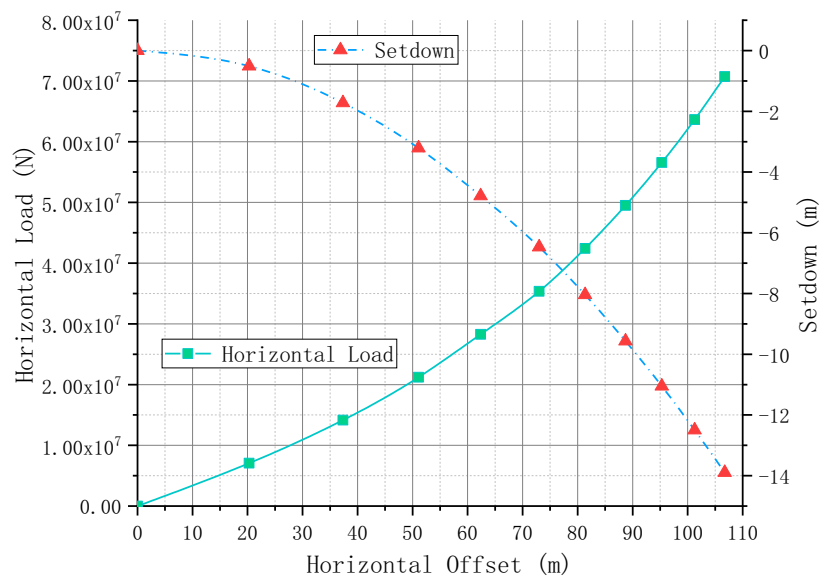

Figure 12 Horizontal stiffness and set-down curve at $45^{\circ}$ (Yu et al., 2019)

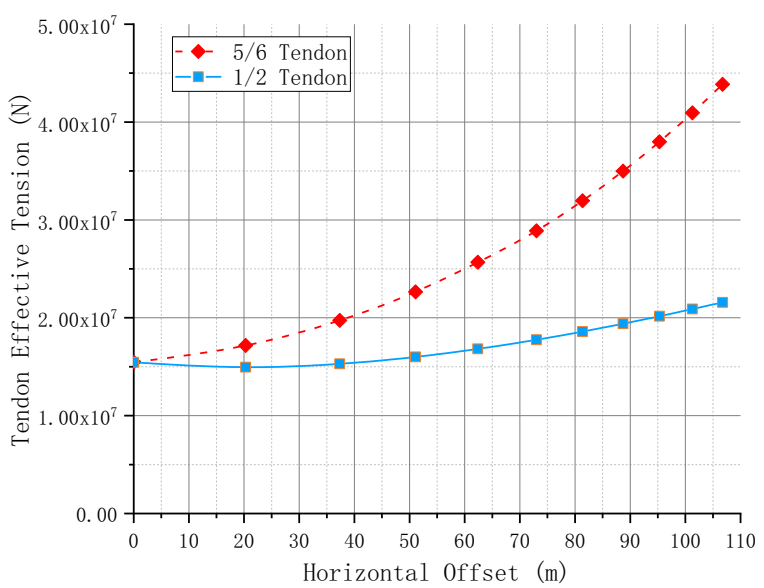

Figure 13 Tendon tension curve at $45^{\circ}$ (Yu et al., 2019)

\subsection{Free Decay Test}

To acquire the structural natural periods in each DOF, a series of free decay tests are performed. The displacement decay curves are plotted in Figure 14.
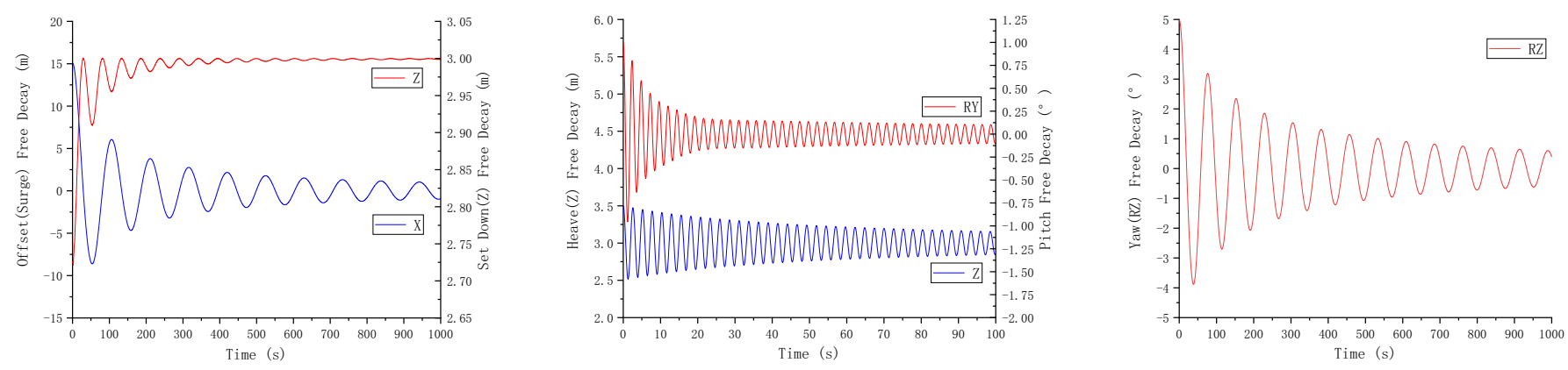

Figure 14 Linear and angular displacements in free decay 
Table 5 Natural Period and Damping Ratio of the Mooring System

\begin{tabular}{|l|l|l|l|}
\hline DOF & $\begin{array}{l}\text { Damping } \\
\text { Ratio }\end{array}$ & $\begin{array}{l}\text { Natural } \\
\text { Period }\end{array}$ & $\begin{array}{l}\text { Initial Offset } \\
\text { or Rotation } \\
\text { of the hull }\end{array}$ \\
\hline Surge/Sway & $3.83 \%$ & $104.52 \mathrm{~s}$ & $15.0 \mathrm{~m}$ \\
\hline Heave & $0.22 \%$ & $2.60 \mathrm{~s}$ & $0.5 \mathrm{~m}$ \\
\hline Pitch/Roll & $0.20 \%$ & $2.34 \mathrm{~s}$ & $1.0^{\circ}$ \\
\hline Yaw & $2.43 \%$ & $76.11 \mathrm{~s}$ & $5.0^{\circ}$ \\
\hline
\end{tabular}

Table 5 shows the natural periods and damping ratios of the mooring system, which are obtained from the decay curves in 6 DOFs. The methodology used to obtain the damping ratios is inspired by Tang (2002). Due to the different mooring stiffness in 6 DOFs, natural periods will be different consequently.

\subsection{Tensioner Test for $\mathbf{4}$ Combined Cylinders}

In the tensioner tests for 4 combined cylinders, 2 types of tests are performed for the vertical tension of the tensioner versus the stroke and the lateral restoring force versus the lateral and vertical offset of the tension ring, respectively, results shown in Figure 15 and Figure 16 . The tension ring stroke, or the riser stroke, is defined as the length of the tension joint between the guide rollers and the tension ring. Due to the real-time angles between the tension joint and cylinders, each tension ring stroke is corresponded to a certain piston stroke of the cylinder. It is represented by the top and bottom coordinate axes. The corresponding relationship between the combined tension and the piston tension is also shown in Figure 15.

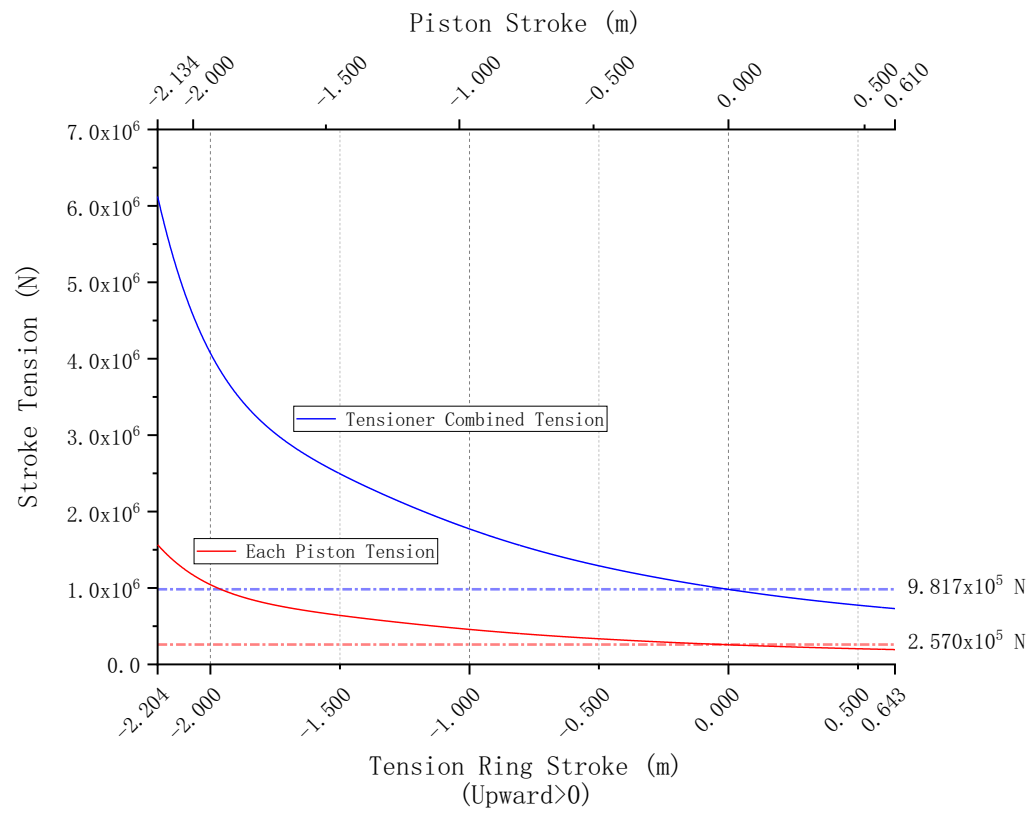

Figure 15 Tensions based on strokes

The second test is performed to obtain the restoring force on the tension ring provided by the tensioner when the tension ring has a horizontal offset from its equilibrium position at different heights. All the displacements and forces are measured based on the tensioner LSA. 


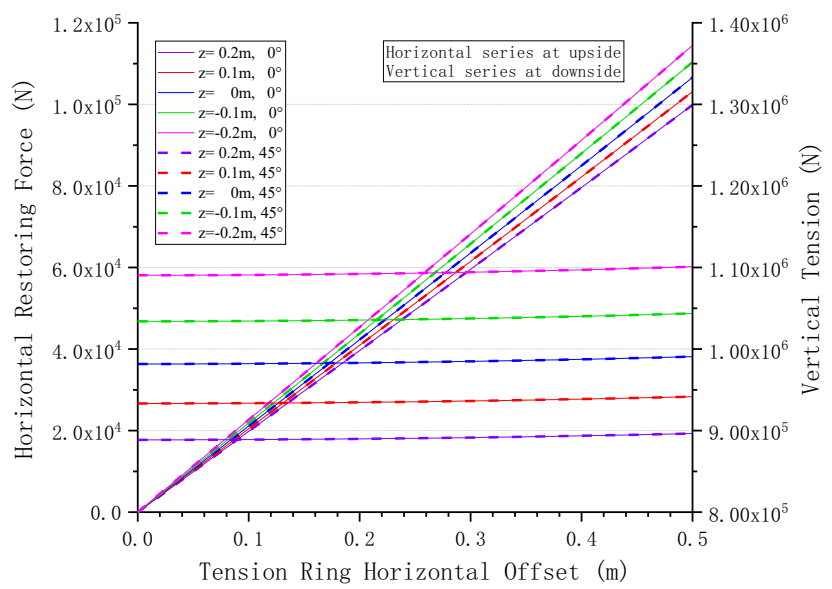

Figure 16 Horizontal and vertical restoring force against the offset of the tension ring

\section{Results and Discussions}

The local failure of the HPT is analyzed by using the fully coupled TLP-TTR model under the calm sea, regular waves, and extreme sea states combined with irregular waves, winds, and currents. In the calm sea, the local failure is simulated to reveal the pure structural response of the coupled model without environmental loads. 3 regular wave conditions are selected with a combination of different wave periods and directions to demonstrate the response regularity. 2 extreme sea states in opposite directions are tested to generate the results under more realistic situations. The configuration of the fully coupled model is quite complicated, which includes several rigid bodies, thus $0.02 \mathrm{~s}$ is selected to be the sufficiently short time step when running all the calculations. The results of the numerical simulation are exported by an interval of $0.1 \mathrm{~s}$. Total duration of each time history is $1000 \mathrm{~s}$ to make the initial condition effect and the transient response from the time the tensioner fails decay adequately.

The modeling and calculation are achieved on a computer workstation which has an 8-core processor and 12 GB memory. The function of parallel processing is launched in AQWA. The memory usage is about $80 \%$ when the model is loaded in the software. For the frequency domain analysis, about 2 hours are used up and it is needed to run only one time for the same wet surface model. For the time domain analysis, about 1 hour is used up to calculate each load case.

\subsection{Calm Sea}

In the calm sea, the time histories of the tensions acted on different cylinders of $R_{1}$ are shown in Figure 18 , and the time histories of their strokes are demonstrated in Figure 19. The deflection curves of $R_{1}$ at the different times after the failure are indicated in Figure 20 . The platform and all the TTRs keep their equilibrium position all the time before the local failure of the tensioner occurs. Therefore, if no Stribeck friction is considered, all the strokes of the cylinders do not change from $0 \mathrm{~m}$ to other values, and all the tension of the cylinders keep at $257 \mathrm{kN}$. This is an idealized situation for a deep-water floating platform, but it is still of high significance when discussing structural failure.

Assuming Cylinder 1 of the tensioner fails at $500 \mathrm{~s}$, different transient responses appear immediately on the different cylinders within a short period of time about 2-3 seconds. Apart the tension of Cylinder 1 decreases to a valley at $36.69 \mathrm{~N}$, the tension of Cylinder 2 and Cylinder 4 increase to a high peak at $297.6 \mathrm{kN}$, and the tension of Cylinder 3 increases to another high peak at $291.6 \mathrm{kN}$. Cylinder 1 sees an $85.7 \%$ decrease, Cylinder $2 / 4$ see a $15.8 \%$ increase, and Cylinder 3 sees a $13.5 \%$ increase. Cylinder $2 / 4$ see the almost same response after the failure occurs, demonstrated by their nearly overlapping tension curves and stroke curves each other. It means that the tension joint of $R_{1}$ swings and tilts within the plane of $45^{\circ}$ (the plane formed by the axes of Cylinder 1 and Cylinder 3) around the guide rollers. Different from Cylinder 2/3/4, after the first valley appears, there are a second valley at $4.79 \mathrm{kN}$ and a third valley at $-2.92 \mathrm{kN}$ of the tension on Cylinder 1 . The third valley is even lower than the first valley at $36.69 \mathrm{~N}$. The minus value of the third valley means that there is only tension from the viscosity after the tension based on the stroke is lost, and the piston of Cylinder 1 rises in a distance again after it drops suddenly. The first valley comes from the sudden failure, and then the stroke velocity increases from zero as the piston drops. The second valley is caused by the tension redistribution of the 3 remaining cylinders. However, the tension redistribution is brought about by the transient interactions between different cylinders because the 3 cylinders do not yet get steady again. The third valley refers to the maximum stroke velocity in the upward direction. The reason why the piston of Cylinder 1 rises again is that it dropped over the equilibrium position in the steady stage. 
As for the strokes of the cylinders, all the cylinders have extensions after the failure. Cylinder 1 sees a valley of its stroke at $-0.192 \mathrm{~m}$. Cylinder 2 and Cylinder 4 see a valley of $-0.102 \mathrm{~m}$. Cylinder 3 sees a valley of $-0.077 \mathrm{~m}$. Different from Cylinder 1 and Cylinder 3 , after the first valley appears, there is a second valley of the stroke of Cylinder $2 / 4$ at $-0.110 \mathrm{~m}$. The second valley is also lower than the first valley at $-0.102 \mathrm{~m}$, because Cylinder 2 and Cylinder 4 are not released completely at the first valley due to the transient interactions between different cylinders. The behaviors of each cylinder following the failure immediately are summarized as that: firstly, all the cylinders drop down because the 3 remaining cylinders cannot provide enough tension when they are at the initial equilibrium positions; secondly, Cylinder 2/4 spring back when they drop down over the new equilibrium positions, and the motion of Cylinder 3 can be regarded as a combination of the spring-back and the compression due to the swing of the tension joint, and Cylinder 1 continues to drop further and is stretched longer by the swing of the tension joint; thirdly, Cylinder 2/4 swing back slightly as Cylinder 1 is compressed and Cylinder 3 is stretched again; fourthly, the cylinders find their new equilibrium positions respectively by the damping and decaying; finally, the tensioner gets into a steady stage with a new tilting angle of the tension joint and different strokes of the cylinders. The static response of $R_{1}$ when the system moves to the steady stage is shown again in Figure 17 . The green cylinder representing the tension joint of $R_{1}$ becomes tilting.

In the steady stage after the transient stage, the average tension of Cylinder 1 decreases to zero, the tension of Cylinder 2 and 4 increase to $271.2 \mathrm{kN}$, and the tension of Cylinder 3 increases to $261.0 \mathrm{kN}$. There are a 5.5\% increase on Cylinder 2/4, and a $1.6 \%$ increase on Cylinder 3 . It should be noticed is that, even though the tensioner is in the steady stage, there are still reactions due to a response propagation of the TTR, which can be seen from the intermittent fluctuations on the curves during the steady stage, both on the tension curves and the stroke curves. As the fluctuation appears intermittently, it becomes weaker and weaker. It is an interesting phenomenon which is not easily observed in the responses of the local mooring failure (Yu et al., 2019). Because of its high slenderness ratio, the TTR is like a string tightened between the tensioner and the subsea wellhead. The local failure of the HPT can be regarded as an initial disturbance at the top end of the string, thus a wave will spread from its top end to bottom end, and then it will return and repeat several times until the wave is dissipated by the structural damping and the hydrodynamic damping gradually. The propagation return period is about $18 \mathrm{~s}$, same as the recurrence period of the fluctuation shown on the curves. The propagation procedure can be also shown in the figure of the riser's deflection curves. Figure 21 presents the above propagation procedure by the exported 3D results.

When Stribeck friction is taken into account, the dashed curves are introduced into the figures to demonstrate the responses with the friction and to contrast them with the responses without the friction. Due to the discontinuity of the function in Stribeck friction model, the tension curves of the cylinders are no longer smooth, but fluctuating. Moreover, the stroke curves of the cylinders added some slight and high frequency fluctuations. While being a method to approach the realistic friction situation between the cylinder and piston, it does not include the realistic static friction. The realistic friction combined with the static and sliding frictions is quite complicated and it is difficult to handle so far. In the study, when the piston has an explicit tendency of the relative motion to the cylinder, Stribeck friction model is precise enough to simulate the sliding friction. This is also why the tensioner responses are analyzed without Stribeck friction first. As shown on the tension curves, when the piston descends, Stribeck friction gives the cylinder more tension; when the piston rises, Stribeck friction counteracts some tension on the cylinder. Thus, in the first peaks of Cylinder $2 / 3 / 4$ or in the first valley of Cylinder 1 , all the cylinders see larger peak values of their tension than those without Stribeck friction. Then, when the tension is at a valley, Stribeck friction flattens and gets the curve lower, while the tension is at a peak it also flattens and gets the curve higher. As shown on the stroke curves, Stribeck friction moderates the change of the strokes in the transient stage, so that no such intensive fluctuations appear again. It can be shown in the figure of the riser's deflection curves that Stribeck friction, which leads to the different motion at the top end of the riser string, forming a different time history of the riser response. 


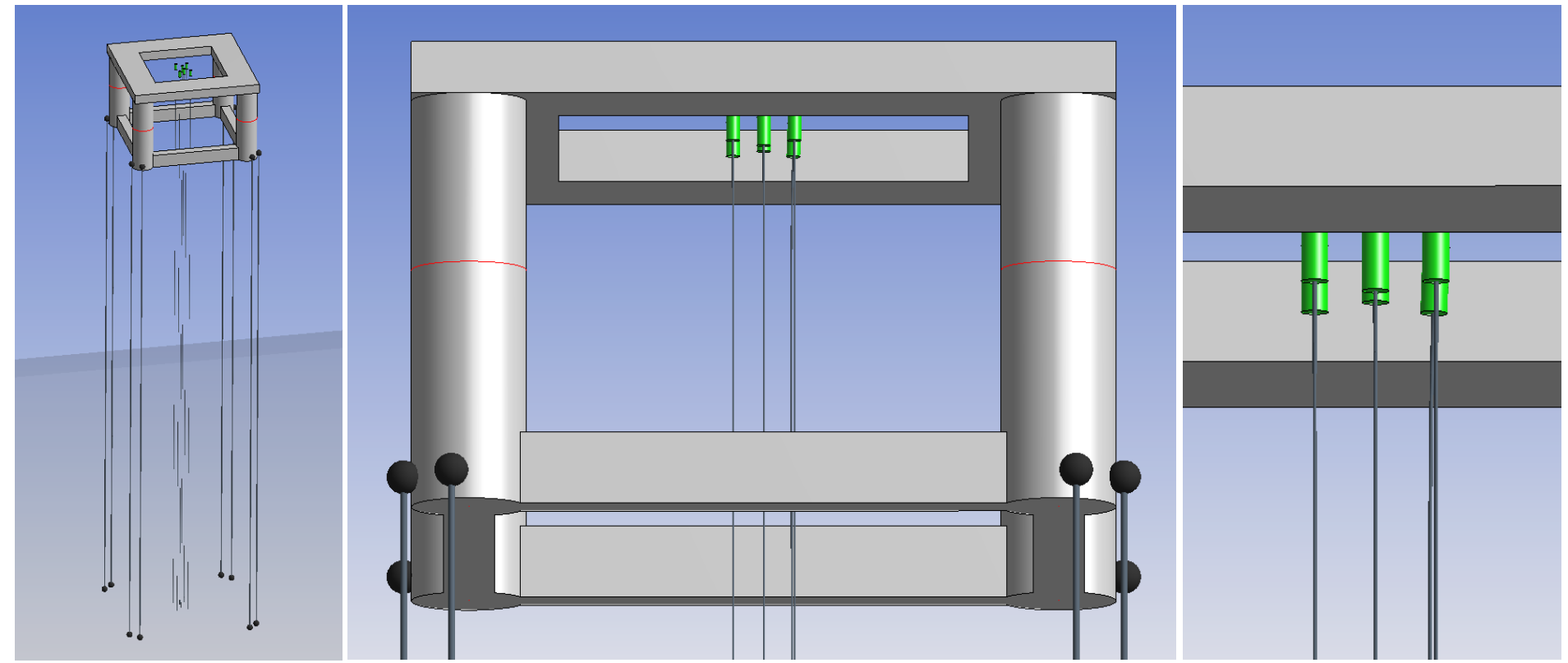

Figure 17 The static response of $R_{1}$ when the system develops into the steady stage again, in calm sea, under the failure of Cylinder 1 


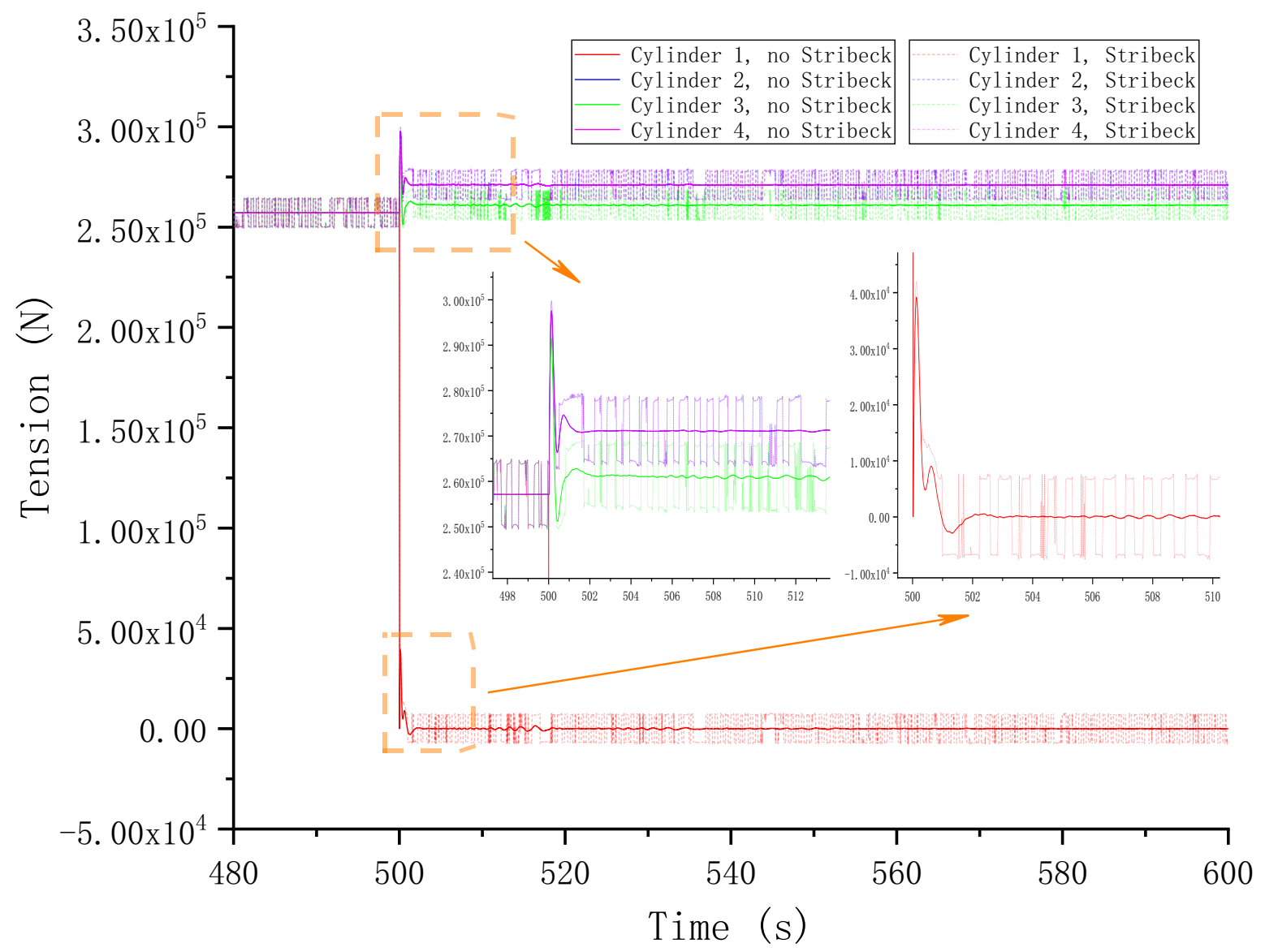

Figure 18 Time histories of tension on the cylinders, in calm sea, under the failure of Cylinder 1 


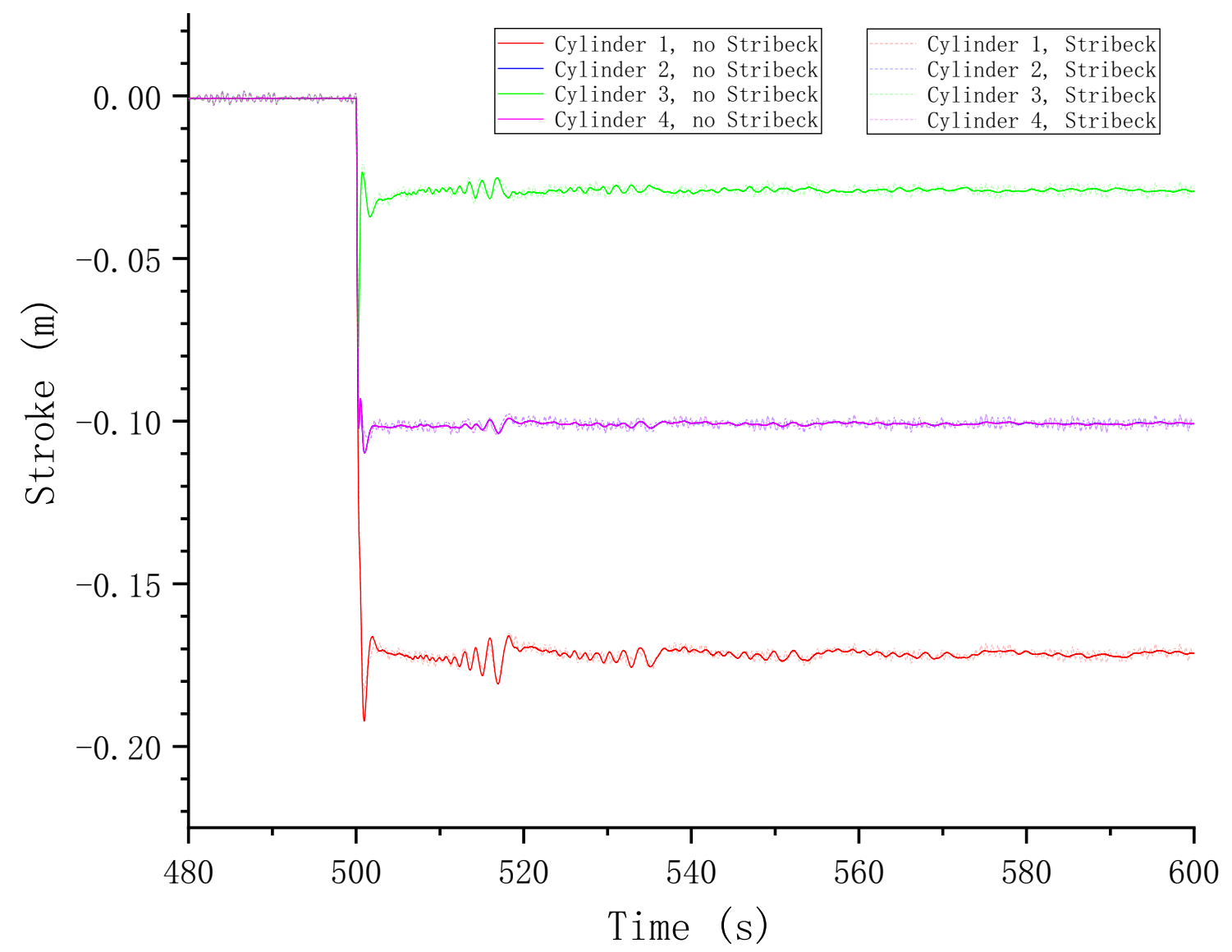

Figure 19 Time histories of strokes of the cylinders, in calm sea, under the failure of Cylinder 1 


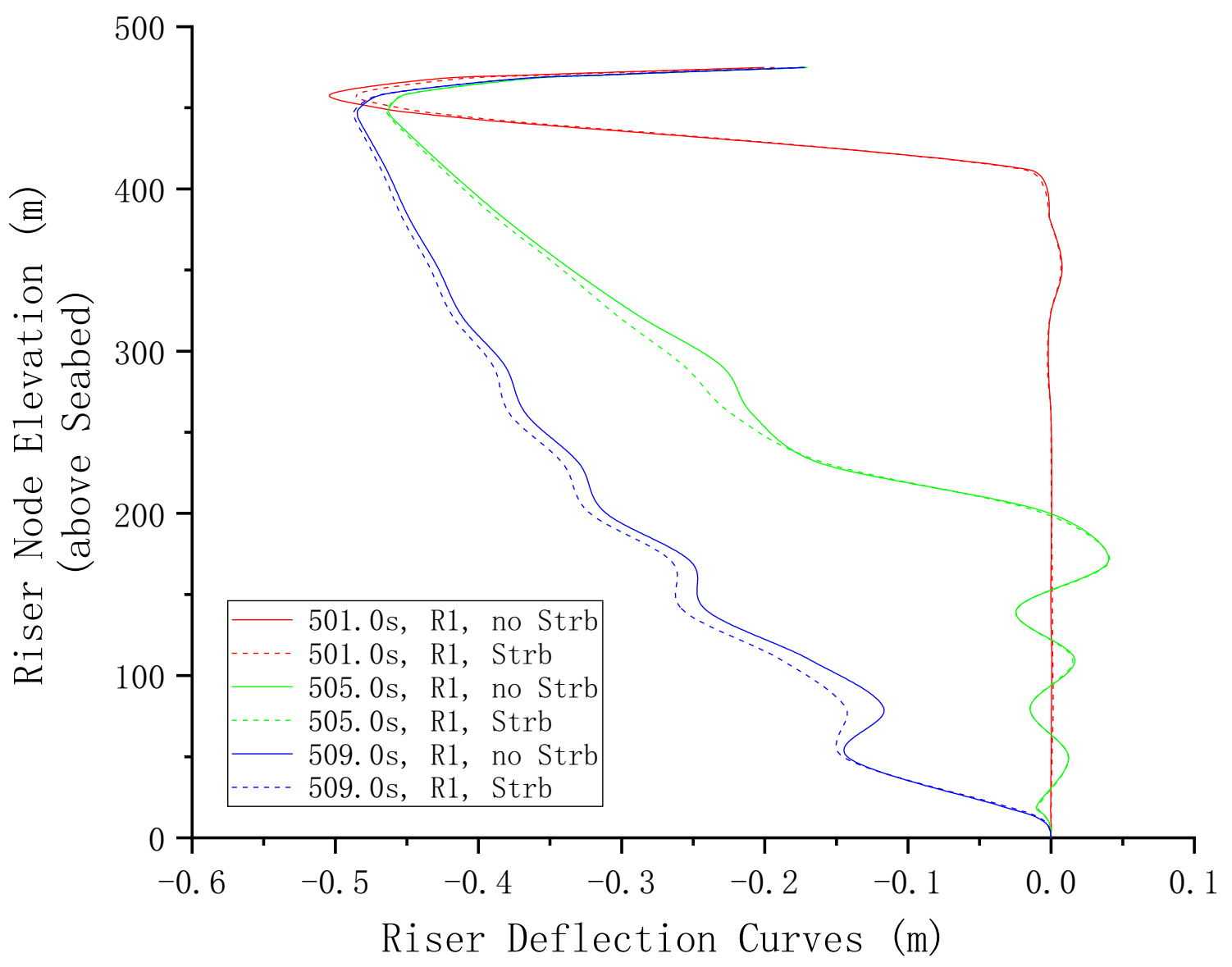

Figure 20 Deflection curves of $R_{1}$ at $501 \mathrm{~s} / 505 \mathrm{~s} / 509 \mathrm{~s}$, in calm sea, under the failure of Cylinder 1 

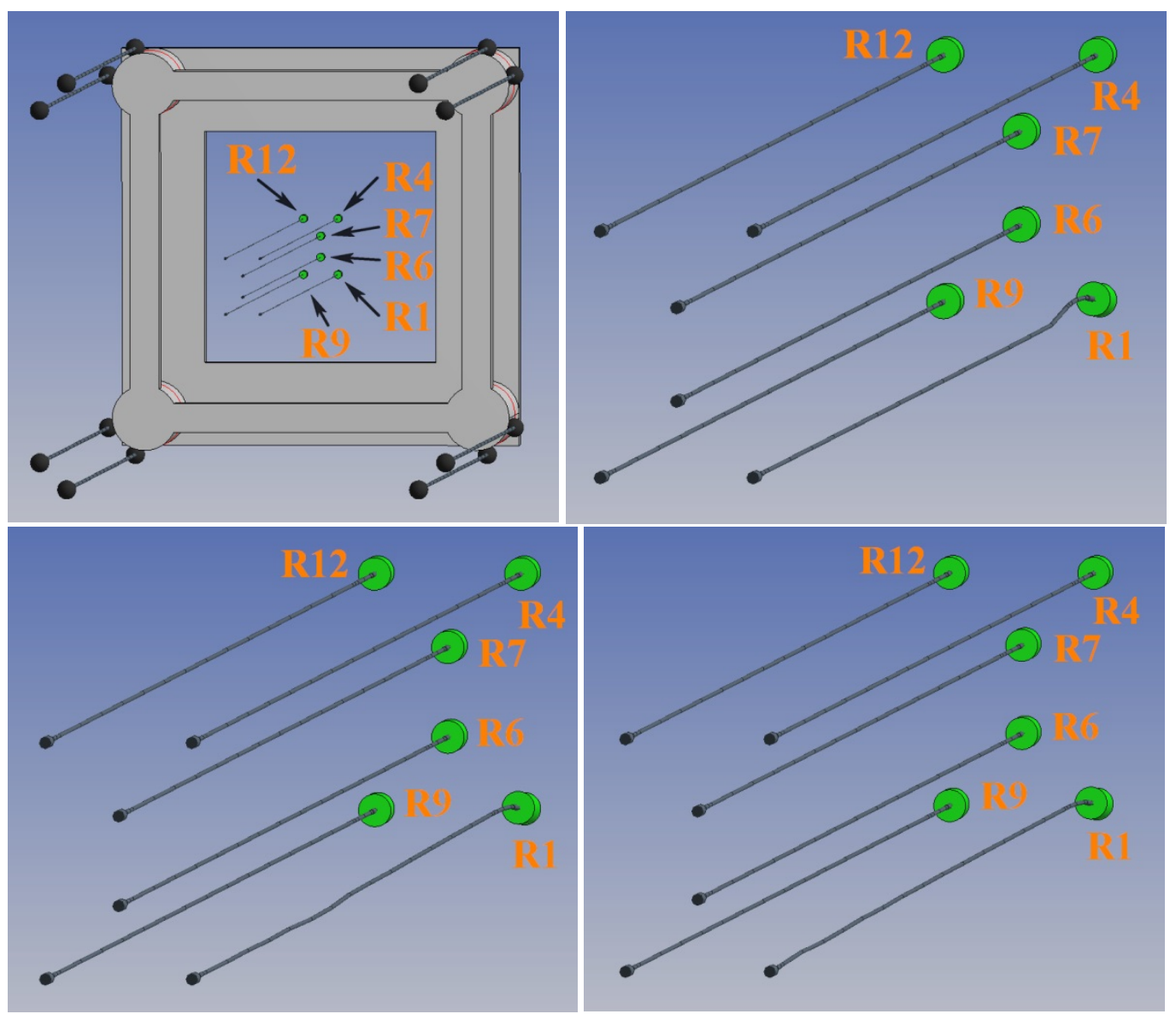

Figure 21 Deflections (axonometric projections) of $R_{1}$ at $501 \mathrm{~s}$ (top right) / $505 \mathrm{~s}$ (lower left) / $509 \mathrm{~s}$ (lower right), in calm sea, under the failure of Cylinder 1

\subsection{Regular Wave}

The basic function of a riser tensioner is to moderate and to adjust the variation of the riser's top tension due to the motions of the platform and the riser under environmental loads. Although as the riser is stretched longer than the initial length, its top tension also increases as if there is no tensioner installed between the production deck and its top end, the top tension does not increase high enough to damage the structure, vice versa. The most typical environmental load acting on a floating platform is the hydrodynamic force from regular waves. To research the responses of the HPT local failure under different regular waves, there are 3 load cases selected with different wave periods and wave directions to make the analysis. Different from the local mooring (tendon) failure of the TLP, because the elevation of the tensioner is much higher than the waterline and it does not touch the water all the time, it will not make a big difference between the responses under the up-wave and down-wave (Yu et al., 2019). Thus, the waves in $180^{\circ}$ and $225^{\circ}$, which are the opposite directions of $0^{\circ}$ and $45^{\circ}$, are not selected in this study. The response results are contrasted between the tensioner failures without and with Stribeck friction. The former is shown with the solid lines, and the latter is shown with the dashed lines in the following figures.

\section{Wave in $10 \mathrm{~s}, 8 \mathrm{~m}, 0^{\circ}$}

Under the wave in $10 \mathrm{~s}, 8 \mathrm{~m}, 0^{\circ}$, the motion response of the hull and deflections of the risers after the tensioner failure (chosen at a representative instant as a hint) are shown in Figure 22, the time histories of the tensions acted on different cylinders of $R_{1}$ are shown in Figure 23, and the time histories of their strokes are demonstrated in Figure 24. The deflection curves of the failed $R_{1}$ and the healthy $R_{4}$ at $510 \mathrm{~s}$ are indicated in Figure 25 . First of all, divide each time history into 3 sections, named as the healthy stage, transient stage, and steady stage. The first one is from the initial condition effect almost decays to $500 \mathrm{~s}$, when the tensioner of $R_{1}$ is still in the healthy condition. It should be noted that to predict the failure response which begins from a conventional response for the reality, instead of a static response, it is necessary to run the time domain calculation in the healthy stage for a sufficient period of time before the local failure, rather than to make the calculation begin from the failure. The second one is from $500 \mathrm{~s}$, the time Cylinder 1 suddenly loses its tension, to the time it restores the stability, which maybe varies when different load cases are applied. The third one is from the time the tensioner restores to the end, $1000 \mathrm{~s}$. 
In the healthy stage, Cylinder 1 and Cylinder 4 almost have the same responses of the tension and stroke, because of the symmetry of the cylinders in the direction. The same phenomenon also occurs on the Cylinder 2 and Cylinder 3 . Nevertheless, the responses of Cylinder $1 / 4$ and Cylinder $2 / 3$ are quite different, no matter on the tension or on the stroke. There is a higher average tension on Cylinder $1 / 4$ than the average tension on Cylinder $2 / 3$, because under the wave in $0^{\circ}$ the platform has an offset along the X-axis of FRA due to the drift force, and then the tension joint tilts. Correspondingly, Cylinder $1 / 4$ has a much lower average stroke than Cylinder 2/3.

In the transient stage, there is a peak tension of $297.9 \mathrm{kN}$ on Cylinder 2 and there is a peak tension of $292 \mathrm{kN}$ on Cylinder 3 and 4 . These tension peaks are higher than the maximum tension of $275.7 \mathrm{kN}$ on the cylinders in the healthy stage. Thus, the local failure of the HPT can make the cylinders experience an impact of the higher tension than that when the HPT is healthy. The tension on Cylinder 1 decreases abruptly and it sees a valley of $-1502 \mathrm{~N}$. This is caused by its upward stroke velocity. On the strokes, all the pistons of the cylinders drop down within an instant after the local failure. Then, Cylinder 1 sees a spring-back point at -0.127 m; Cylinder 2 sees a spring-back point at -0.099 m; Cylinder 3 and Cylinder 4 see a spring-back point at $-0.072 \mathrm{~m}$.

In the steady stage, because of the asymmetry of the cylinders along the wave direction, the responses of Cylinder 2 and Cylinder 3 are no longer the same. Compared with the transient response of the HPT, the steady response is more obvious. The tensions on all the remaining cylinders increase by different extents, because the 3 remaining cylinders begin to share the tension on the TTR which is shared initially by 4 cylinders. Before the failure, the variation range of the tension on of Cylinder 4 is from $251.9 \mathrm{kN}$ to $275.7 \mathrm{kN}$. While after the failure, the range of the tension becomes from $261.2 \mathrm{kN}$ to $297.7 \mathrm{kN}$, a $3.7 \%$ increase of the lower limit and an $8.0 \%$ increase of the upper limit. The peak of $297.7 \mathrm{kN}$ is also higher than the transient peak of $292 \mathrm{kN}$. The shape of the tension curve almost has no change. Before the failure, the variation range of the tension on of Cylinder $2 / 3$ is from $252.0 \mathrm{kN}$ to $261.9 \mathrm{kN}$. While after the failure, the range of the tension of Cylinder 2 rises from $268.6 \mathrm{kN}$ to $275.9 \mathrm{kN}$, a $6.6 \%$ increase of the lower limit and a 5.3\% increase of the upper limit; the range of the tension of Cylinder 3 gets from $258.3 \mathrm{kN}$ to $265.9 \mathrm{kN}$, a $2.5 \%$ increase of the lower limit and a $1.5 \%$ increase of the upper limit. The two curves are almost parallel with each other, while they have a different shape from the curves in the healthy stage. The tension range of the failed Cylinder 1 changes from $-5548 \mathrm{~N}$ to $4173 \mathrm{~N}$, which originated from the viscosity friction.

In the steady stage, two groups of the overlapped stroke curves prior to the failure separate apart respectively into 4 different stroke curves. Before the failure, the stroke of Cylinder $1 / 4$ varies from $-0.127 \mathrm{~m}$ to $0.036 \mathrm{~m}$, while the stroke of Cylinder 1 varies from $-0.339 \mathrm{~m}$ to $-0.105 \mathrm{~m}$, and the stroke of Cylinder 4 varies from $-0.268 \mathrm{~m}$ to $-0.035 \mathrm{~m}$ after the failure. Before the failure, the stroke of Cylinder $2 / 3$ varies from $-0.032 \mathrm{~m}$ to $0.035 \mathrm{~m}$, while the stroke of Cylinder 2 varies from $-0.131 \mathrm{~m}$ to $-0.087 \mathrm{~m}$, and the stroke of Cylinder 3 varies from $-0.061 \mathrm{~m}$ to $-0.013 \mathrm{~m}$ after the failure. The curves of Cylinder 1/4 are almost parallel with each other, and the curves of Cylinder $2 / 3$ are almost parallel, too. The responses in the transient stage and the steady stage can be regarded as the approximate superposition of the response after the local failure in the calm sea and the healthy response in regular waves. Due to such short duration of the transient stage, there is not enough time for the wave with a $10 \mathrm{~s}$ period to influence the transient response. Thus the time history of the tension joint tilting and swinging in the diagonal are almost the same as the calm sea condition. Then the tilted TTR swings in the wave direction as the wave frequency. However, the transient response propagation does not appear under the regular wave, which is shown in the calm sea, due to changing of the tension level in the TTR.

As for the riser response, it is proved in the figure of the risers' deflection curves that it is necessary to model the failed riser and the other healthy risers respectively. They have obviously different riser responses. As shown in Figure 25 , at $510.0 \mathrm{~s}, R_{1}$ with its tensioner failure is much slacker than the healthy $R_{4}$, because the elevation of the tension ring declines after the cylinder strokes are extended as the tension in Cylinder 1 is lost. The slacker riser has lower tension. Note that in the riser array, all the healthy risers should have the nearly synchronous response, because they are in close proximity to each other and they are under the almost same loads, unless the platform inclines obviously under some special situations e.g. the tendon failure (Yu et al., 2019) or the riser interference occurs. Thus, the response of the healthy $R_{4}$ can also represent those of other healthy risers. Only their deformations along the direction of wave are presented. In the figure, the wave comes from the left and goes to the right. Although the failed riser has the deformation perpendicular to the wave direction, it is comparatively small and steady because it has nothing to do with the dynamic loads. As shown in Figure 11, $R_{1}$ and $R_{4}$ are in the same column of the riser array. Thus their deflection curves should be overlapped, if the tensioner failure does not occur. The deflection curve of the healthy riser is not only attributed to its gravity and buoyancy, but also to the dynamic loads, such as the wave load, the riser's inertial load, and the tension load. In addition, the most upper element node should satisfy the boundary condition at the tension ring. Besides its deflection, the abscissa includes the offset of the hull, thus the deflection curve is inclined. When Cylinder 1 of $R_{1}$ fails, the tilt of the tension joint causes a bending on the top end of the curve of $R_{1}$, and the curve end of $R_{1}$ is on the left of the curve end of $R_{4}$. As the tension in $R_{1}$ declines after the failure, the deflection curve of $R_{1}$ has a similar shape and trend with that of $R_{4}$, but $R_{1}$ has larger amplitude. It should be noted that the horizontal and vertical coordinates are in different scales, therefore the shapes of risers have some distortion, but their values are correct. Comparatively with the tensioner failure, Stribeck friction has very weak influence on these curves.

When Stribeck friction participates in the responses of the tensioner, when the piston descends, the tension on the cylinder is higher than that when Stribeck friction is neglected, vice versa. The tension and stroke responses with Stribeck model are demonstrated by the dashed lines in Figure 23 and Figure 24. Compared with the tension response obtained from the calm sea condition, the results are more realistic and significant here, 
because the regular wave load always makes the relative motion of the piston to the cylinder explicit, and the Stribeck friction force is not large enough to change the direction of the relative velocity. Apparently, Stribeck friction can amplify the variation ranges of the tensions on the cylinders, no matter which stage it is in, so that the tension on the TTR is also amplified. Thus, it is necessary to consider the friction when solving the problem. In addition, Stribeck friction does not make the time histories of the strokes evidently different from those without Stribeck friction.
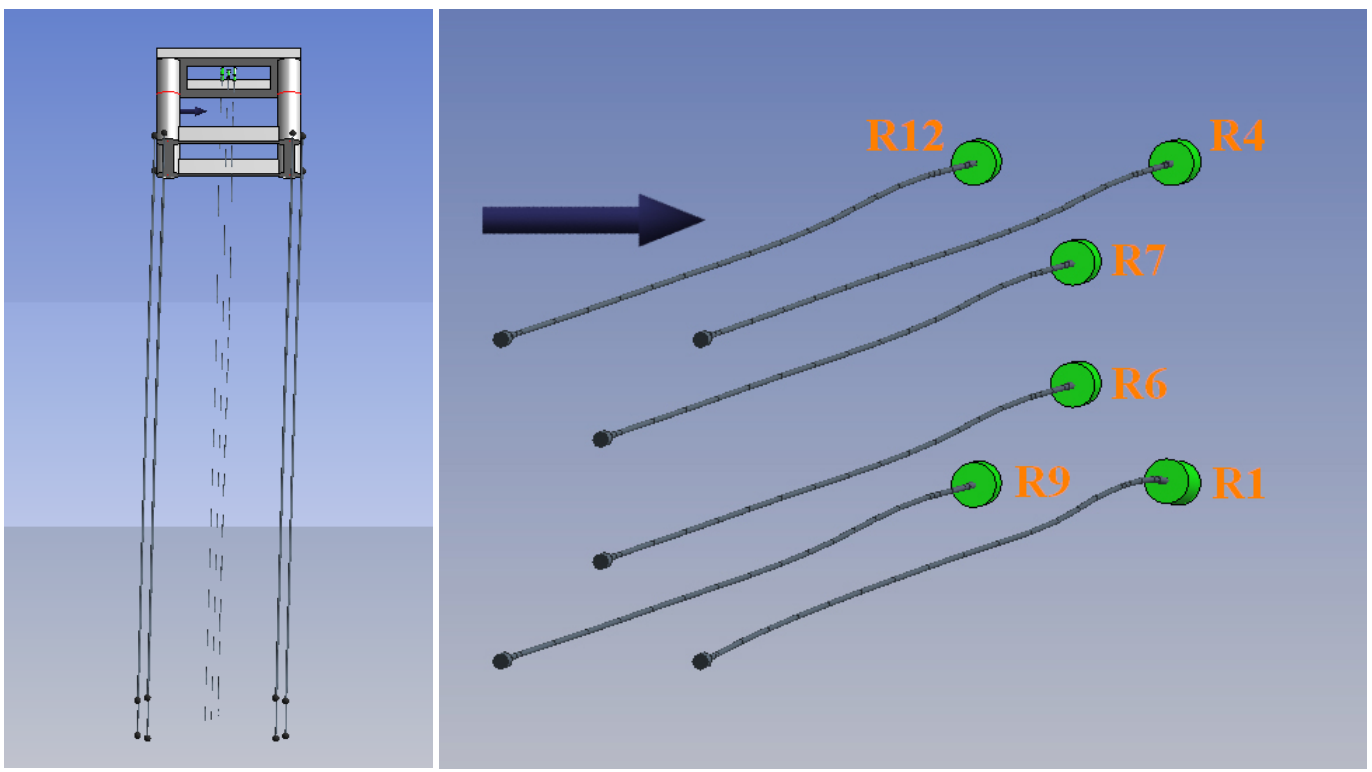

Figure 22 Motion response of the hull and axonometric projections of the risers, wave in $10 \mathrm{~s}, 8 \mathrm{~m}, 0^{\circ}$, under the failure of Cylinder 1 


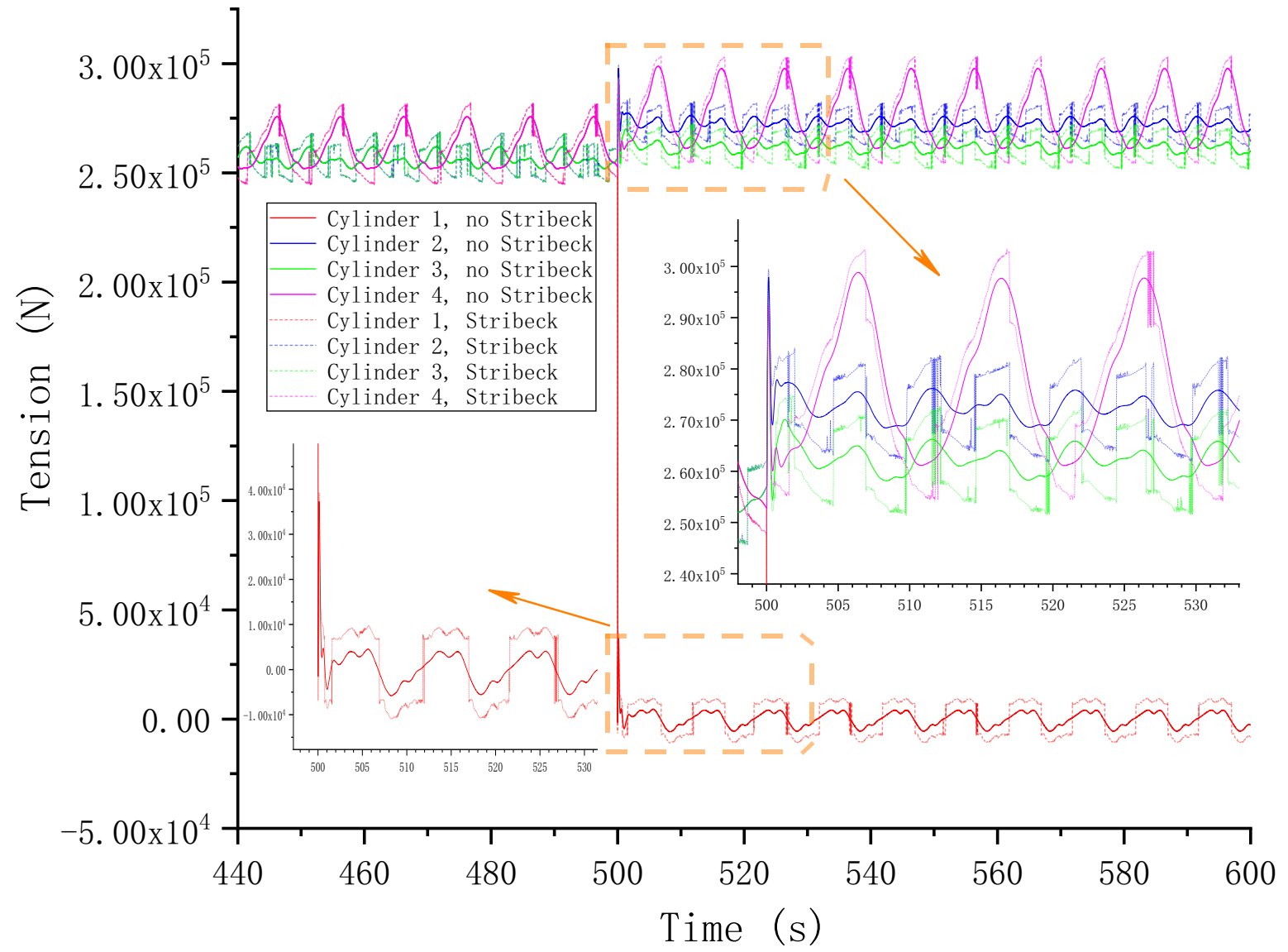

Figure 23 Time histories of tension on the cylinders, wave in $10 \mathrm{~s}, 8 \mathrm{~m}, 0^{\circ}$, under the failure of Cylinder 1 


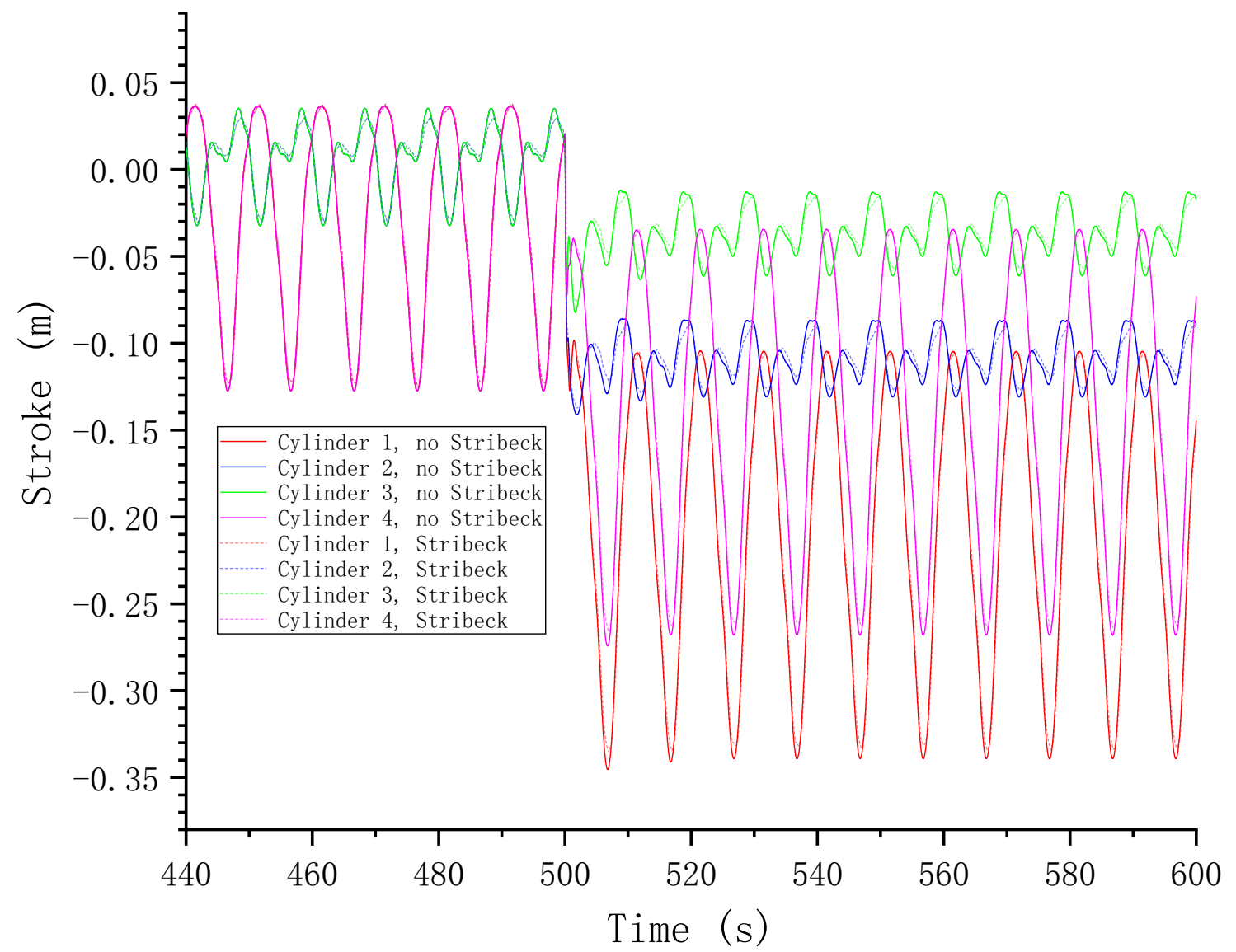

Figure 24 Time histories of strokes of the cylinders, wave in $10 \mathrm{~s}, 8 \mathrm{~m}, 0^{\circ}$, under the failure of Cylinder 1 


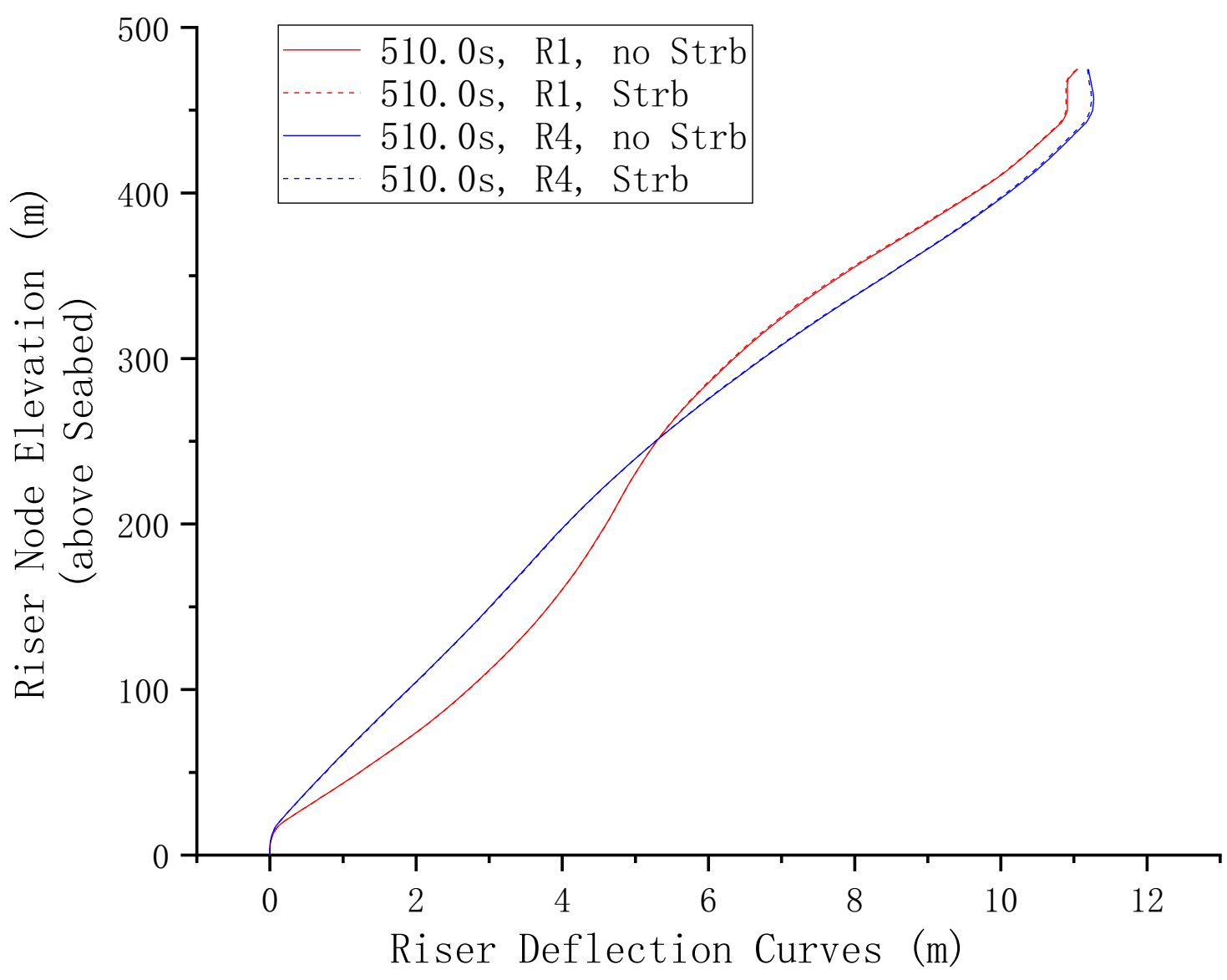

Figure 25 Deflection curves of $R_{1}$ and $R_{4}$ at $510 \mathrm{~s}$, wave in $10 \mathrm{~s}, 8 \mathrm{~m}, 0^{\circ}$, under the failure of Cylinder 1

Wave in $16 \mathrm{~s}, 8 \mathrm{~m}, 0^{\circ}$

Under the wave in $16 \mathrm{~s}, 8 \mathrm{~m}, 0^{\circ}$, the motion response of the hull and deflections of the risers after the tensioner failure (chosen at a representative instant as a hint) are shown in Figure 26, the time histories of the tensions acted on different cylinders of $R_{1}$ are shown in Figure 27, and the time histories of their strokes are demonstrated in Figure 28. The tension and stroke responses with Stribeck model are demonstrated by the dashed lines in Figure 27 and Figure 28. By contrast with the first regular wave condition, there is a variation on the wave period. When the wave period increases, the tension impact on the cylinders correspondingly has a longer influence and a longer time of decaying before the tensioner enters the steady stage, which means the tensioner has a longer transient stage. In the healthy stage, the response shapes of the tension and strokes of the cylinders change significantly as the wave period changes, because the complicated coupled motion of the system, which consists of the hull, tendons, tensioners, and TTRs, is highly sensitive to the load period. While their response shapes in the steady stage are still almost same with those in the healthy stage and the curves move and separate apart to different tension or stroke levels respectively. The transient peak of the tension is over the maximum tension in the subsequent steady stage under the wave condition. As the tensioner failure has a slight influence on the motion of the hull and the TTRs are close to the center of the hull, it can be inferred that the tensioner and riser responses of the other 5 healthy TTRs are almost same with the responses of $R_{1}$ before the failure, and they still last and does not change after the failure. 

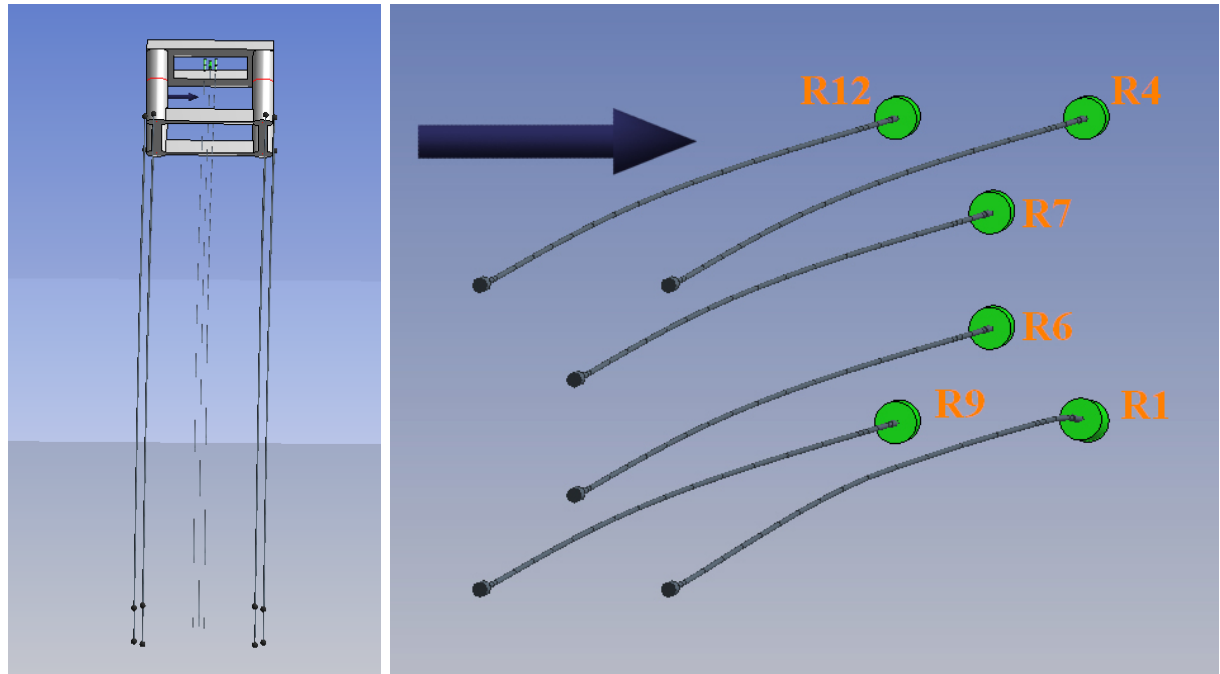

Figure 26 Motion response of the hull and axonometric projections of the risers, wave in $16 \mathrm{~s}, 8 \mathrm{~m}, 0^{\circ}$, under the failure of Cylinder 1 


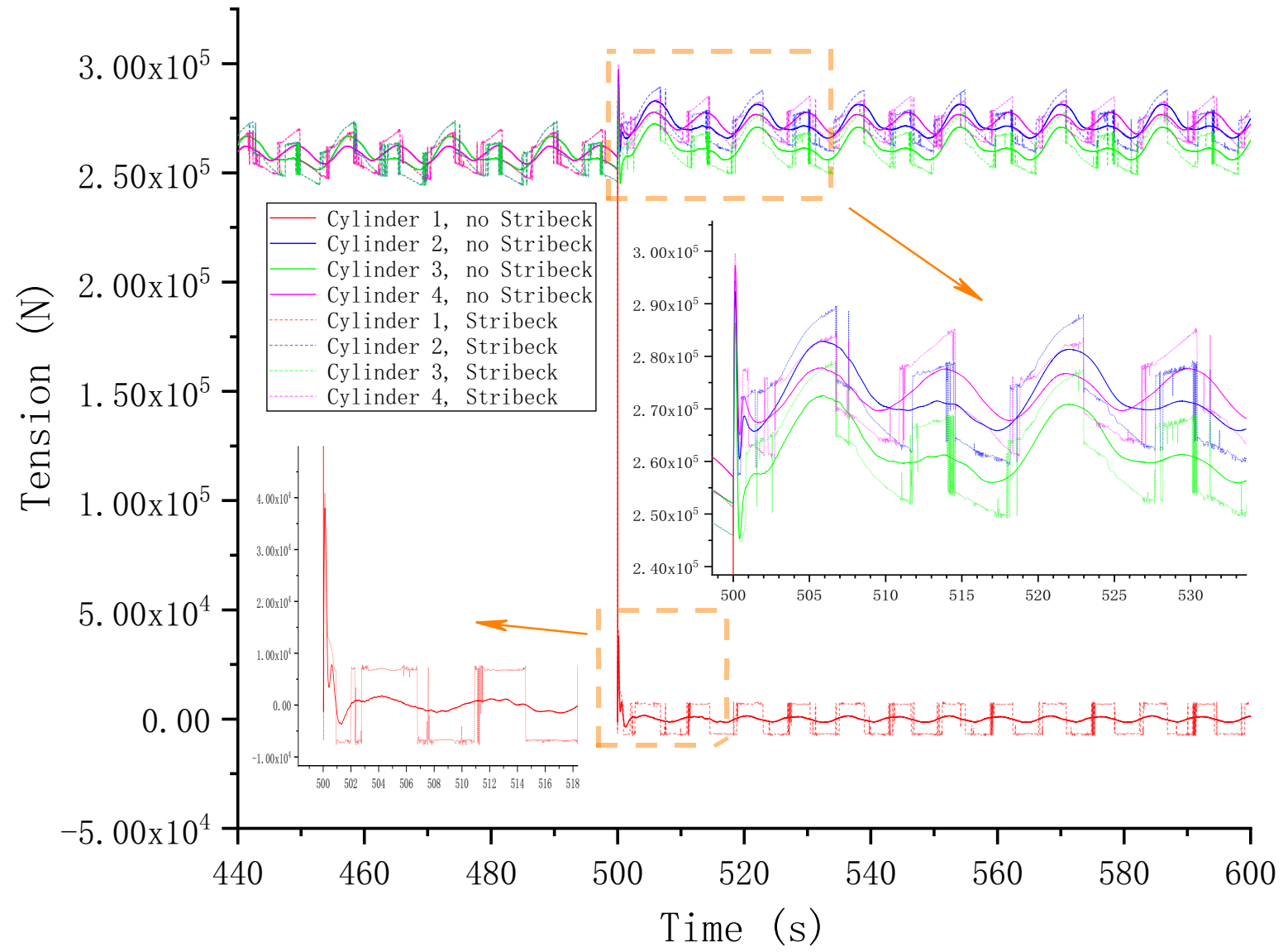

Figure 27 Time histories of tension on the cylinders, wave in $16 \mathrm{~s}, 8 \mathrm{~m}, 0^{\circ}$, under the failure of Cylinder 1 


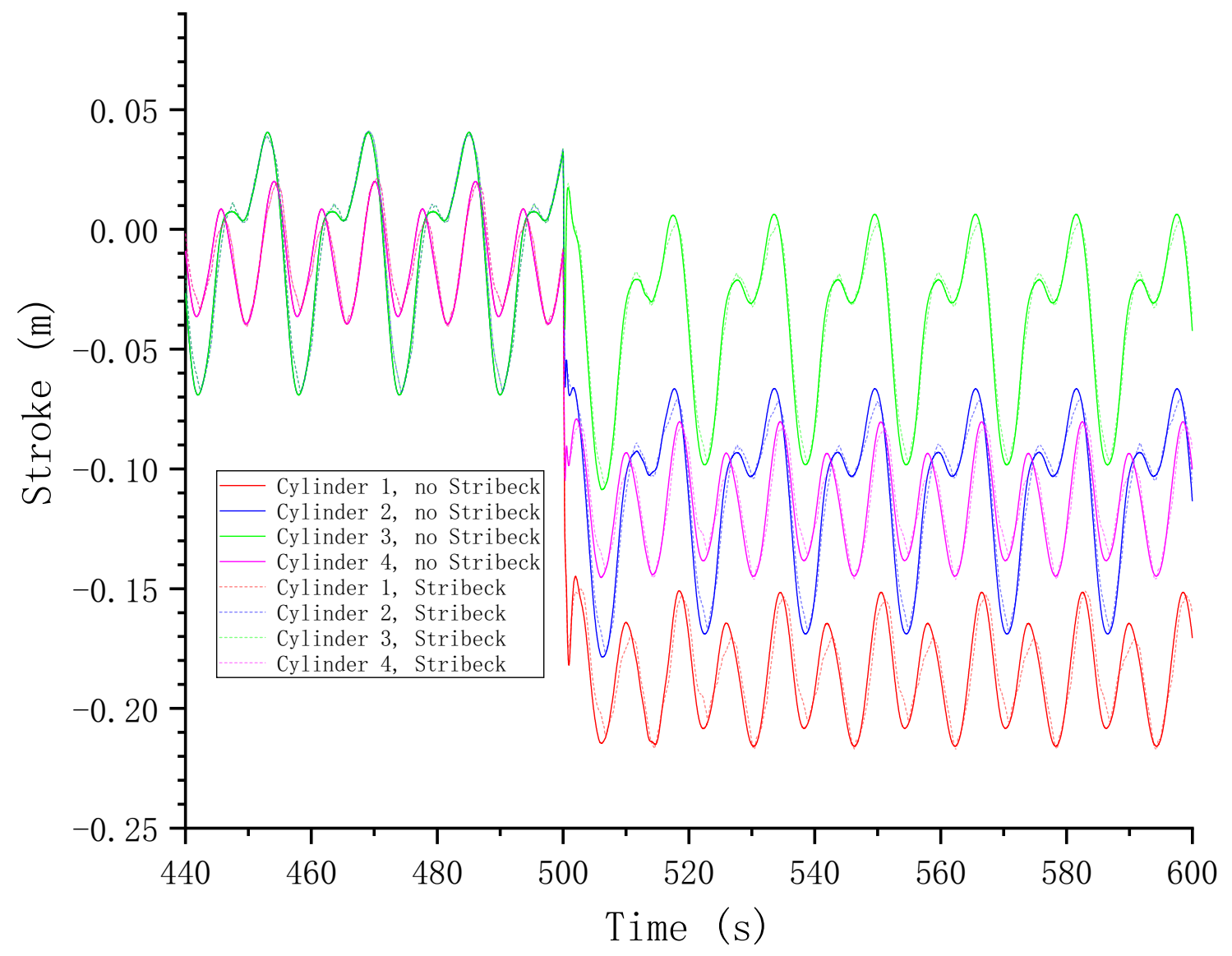

Figure 28 Time histories of strokes of the cylinders, wave in $16 \mathrm{~s}, 8 \mathrm{~m}, 0^{\circ}$, under the failure of Cylinder 1

\section{Wave in $10 \mathrm{~s}, 8 \mathrm{~m}, 45^{\circ}$}

Under the wave in $10 \mathrm{~s}, 8 \mathrm{~m}, 45^{\circ}$, the motion response of the hull and deflections of the risers after the tensioner failure (chosen at a representative instant as a hint) are shown in Figure 29, the time histories of the tensions acted on different cylinders of $R_{1}$ are shown in Figure 30, and the time histories of their strokes are demonstrated in Figure 31. The tension and stroke responses with Stribeck model are demonstrated by the dashed lines in the figures. By contrast, there is another variation on the wave direction. In the case, the direction of the wave load and the axis of the failed Cylinder 1 overlap horizontally, thus there are 3 different responses between Cylinder 1, Cylinder 2/4, and Cylinder 3 in all the stages. The responses of Cylinder 2 and 4 are the same all the time, even though after the tensioner failure. Their tension has an obvious increase subsequently to compensate the loss of Cylinder 1 . In terms of the stroke curves, the tension joint becomes more tilting after the tensioner failure, because the stroke curves of Cylinder 1 and Cylinder 3 no longer have intersections. 


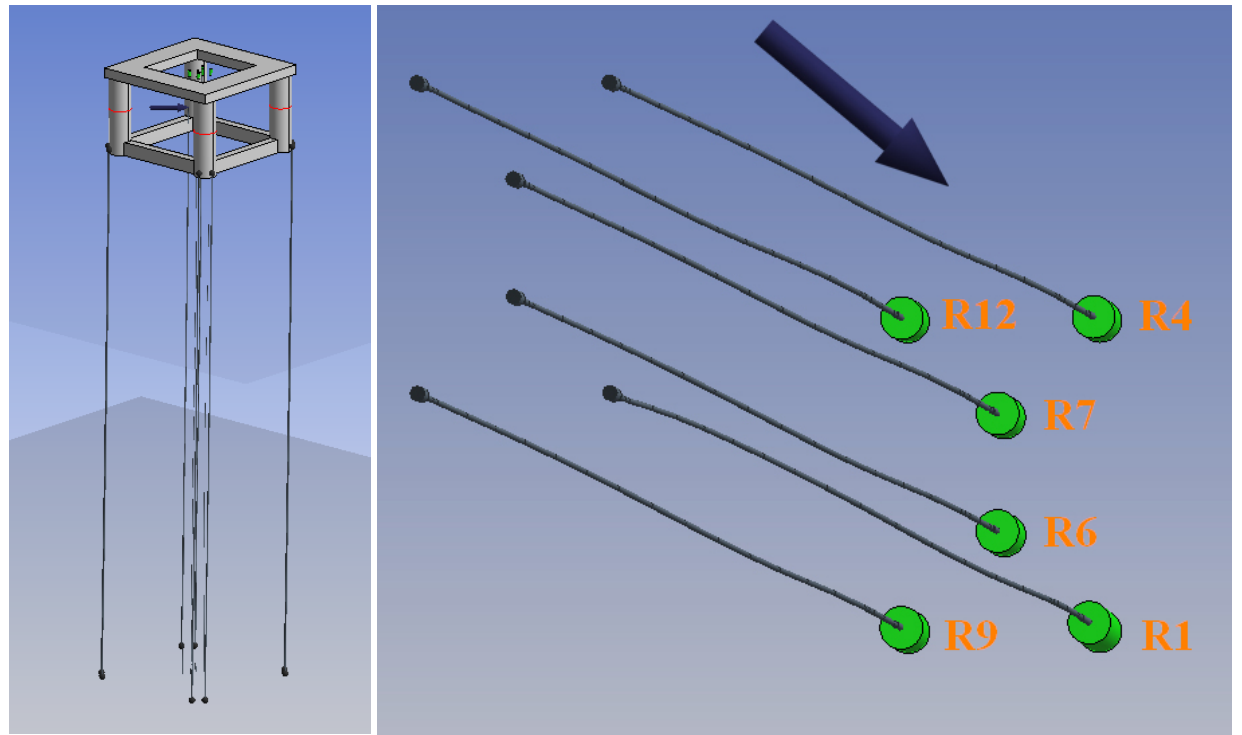

Figure 29 Motion response of the hull and axonometric projections of the risers, wave in $10 \mathrm{~s}, 8 \mathrm{~m}, 45^{\circ}$, under the failure of Cylinder 1 


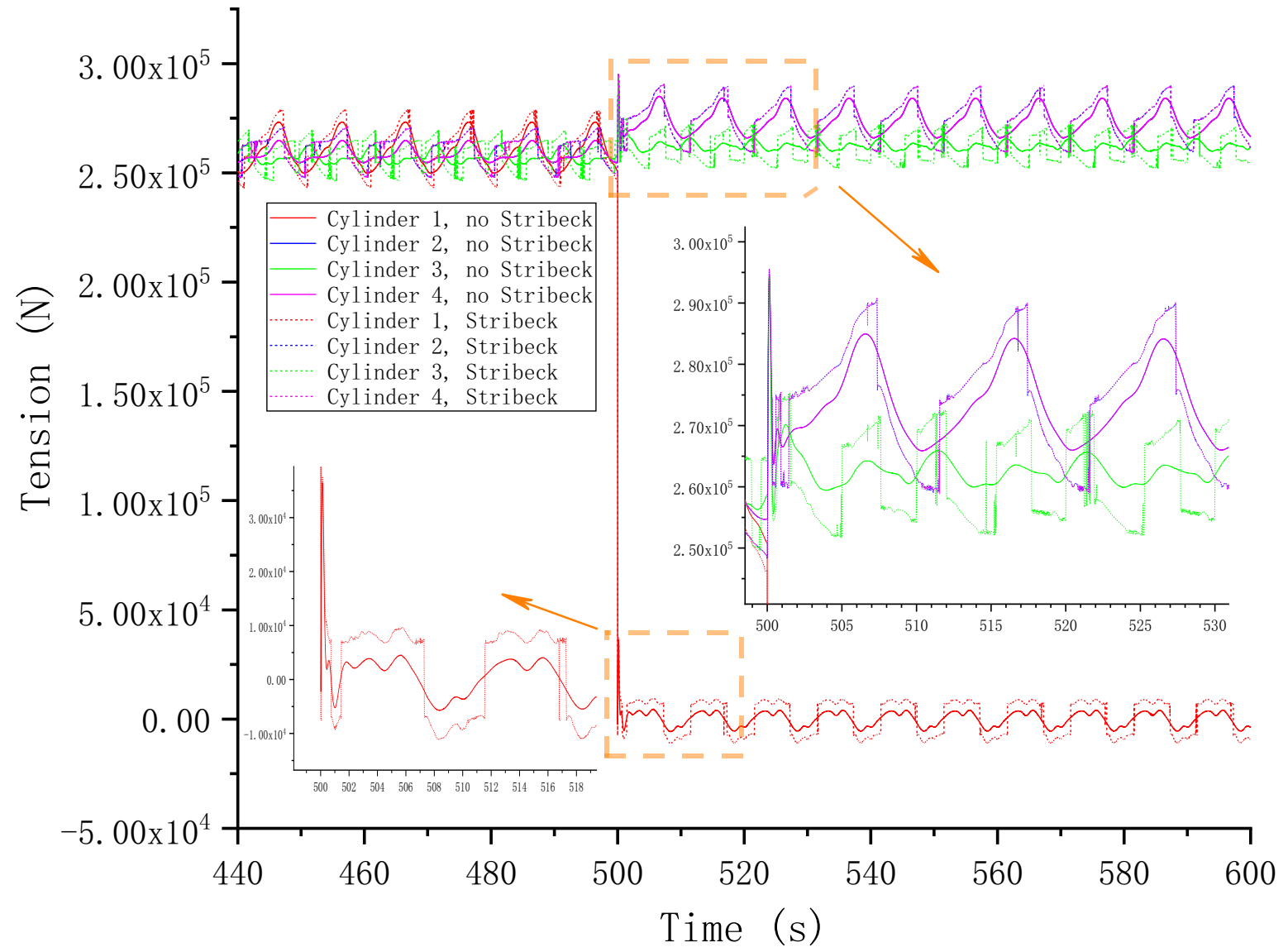

Figure 30 Time histories of tension on the cylinders, wave in $10 \mathrm{~s}, 8 \mathrm{~m}, 45^{\circ}$, under the failure of Cylinder 1 


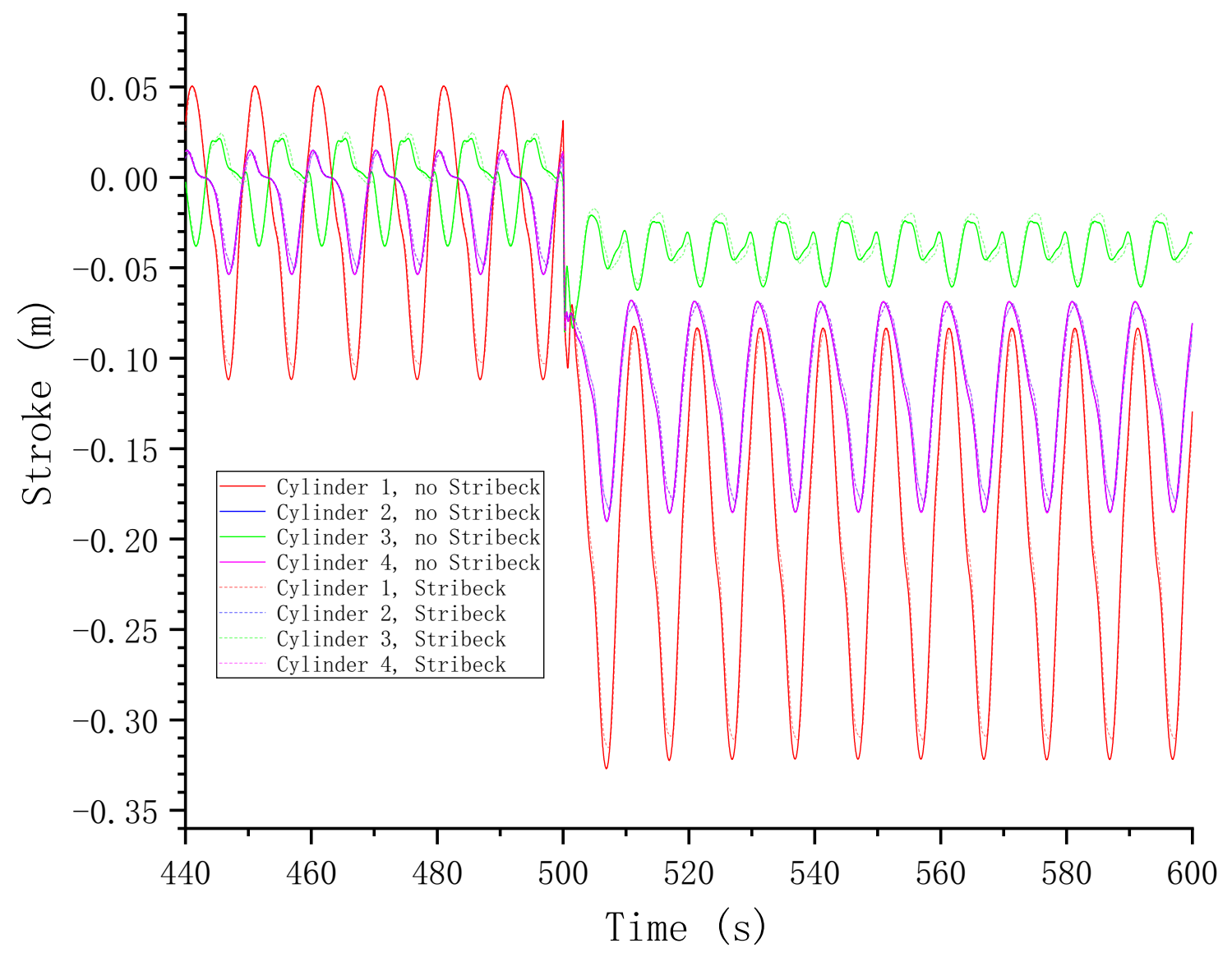

Figure 31 Time histories of strokes of the cylinders, wave in $10 \mathrm{~s}, 8 \mathrm{~m}, 45^{\circ}$, under the failure of Cylinder 1

\subsection{Extreme Sea State}

After finishing the fully coupled model of both the calm sea and the regular wave conditions, it is still necessary to evaluate the responses of the tensioner local failure under the extreme environmental loads. Failure in tensioner is most likely to occur under the extreme sea states when the cylinders and the hydraulic pressure system are working intensively to adjust the top tension of the riser. To consider the relative position between the failed cylinder and the environmental loads, the load direction of $0^{\circ}$ and $45^{\circ}$ are selected respectively when the failed cylinder is at the corner and on the axis of the loads. Though the phase angles in the wave and wind spectrums are distributed randomly when generating the time histories of the wave elevation and wind velocity, to ensure the valid result contrasts, the same time histories are applied to the 2 load cases.

\section{Wave, Current, and Wind in $0^{\circ}$}

Under the wave, current, and wind in $0^{\circ}$, the motion response of the hull and deflections of the risers after the tensioner failure (chosen at a representative instant as a hint) are shown in Figure 32, the time histories of the tensions acted on different cylinders of $R_{1}$ are shown in Figure 33 , and the time histories of their strokes are demonstrated in Figure 34. The tension and stroke responses with Stribeck model are demonstrated by the dashed lines in the figures. Based on the response results output from the time domain analysis, it's logical to assume that the initial condition effect no longer exists after the 200-th second when valid healthy stage begins. In the healthy stage, the tension of cylinders varies from $239 \mathrm{kN}$ to $389.8 \mathrm{kN}$, which is $93.0 \%$ to $151.7 \%$ of the cylinder's initial pretension. In the later steady stage, the tension of cylinders sees an increase and it varies from $256 \mathrm{kN}$ to $522.3 \mathrm{kN}$, which is $99.6 \%$ to $203.2 \%$ of the initial pretension. Between the two stages, in the transient stage, there are only small tension peaks of the cylinders: for Cylinder 4 at $336.2 \mathrm{kN}$, for Cylinder 2 at $329.4 \mathrm{kN}$, and for Cylinder 3 at 322 kN. These tension peaks are still much lower than the peaks appeared in the subsequent stage. With regard to the strokes of the cylinders, in the healthy stage, they vary from $0.674 \mathrm{~m}$ to $0.083 \mathrm{~m}$. In the steady stage, they drop down and range from $-1.192 \mathrm{~m}$ to $-0.032 \mathrm{~m}$. No obvious stroke peaks of the cylinders are 
observed in the figure, which means no explicit transient stage of the stroke responses under the extreme sea state. Different from the responses under the calm sea and regular wave conditions, as the environmental loads are intensified, the transient response of the tensioner is slighter ad slighter. This is attributed to the changing of the tension level in the HPT. More the environmental loads are intensified, higher tension level the HPT has. The responses in the transient stage and the steady stage also can be regarded as the approximate superposition of the response after the local failure in the calm sea and the healthy response in the wave, current, and wind. When Stribeck friction is considered in the simulation, the same features with the tension time histories under regular waves are shown under the extreme environmental conditions. Compared with the curves under the calm sea, no tension curves fluctuate repeatedly, which means that Stribeck friction model is also available for the extreme sea states.
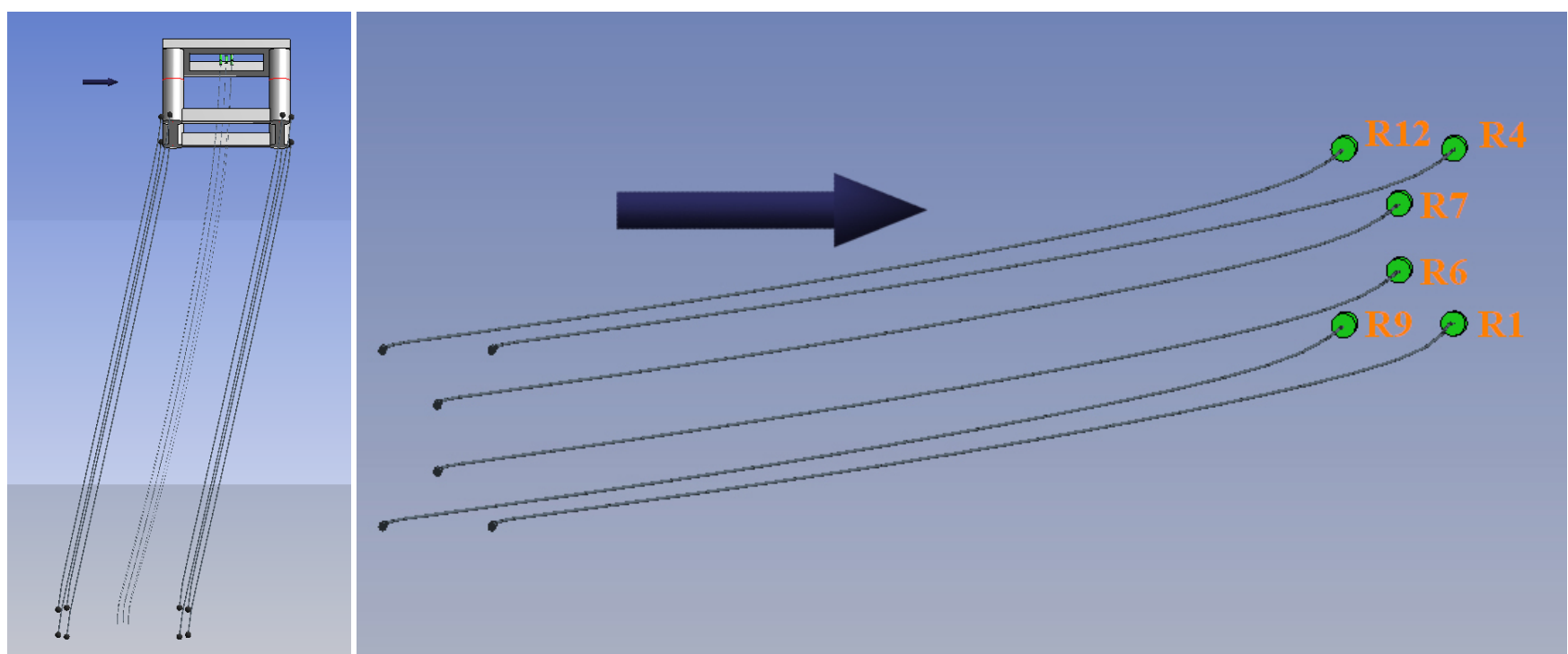

Figure 32 Motion response of the hull and axonometric projections of the risers, wave, current, and wind in $0^{\circ}$, under the failure of Cylinder 1 


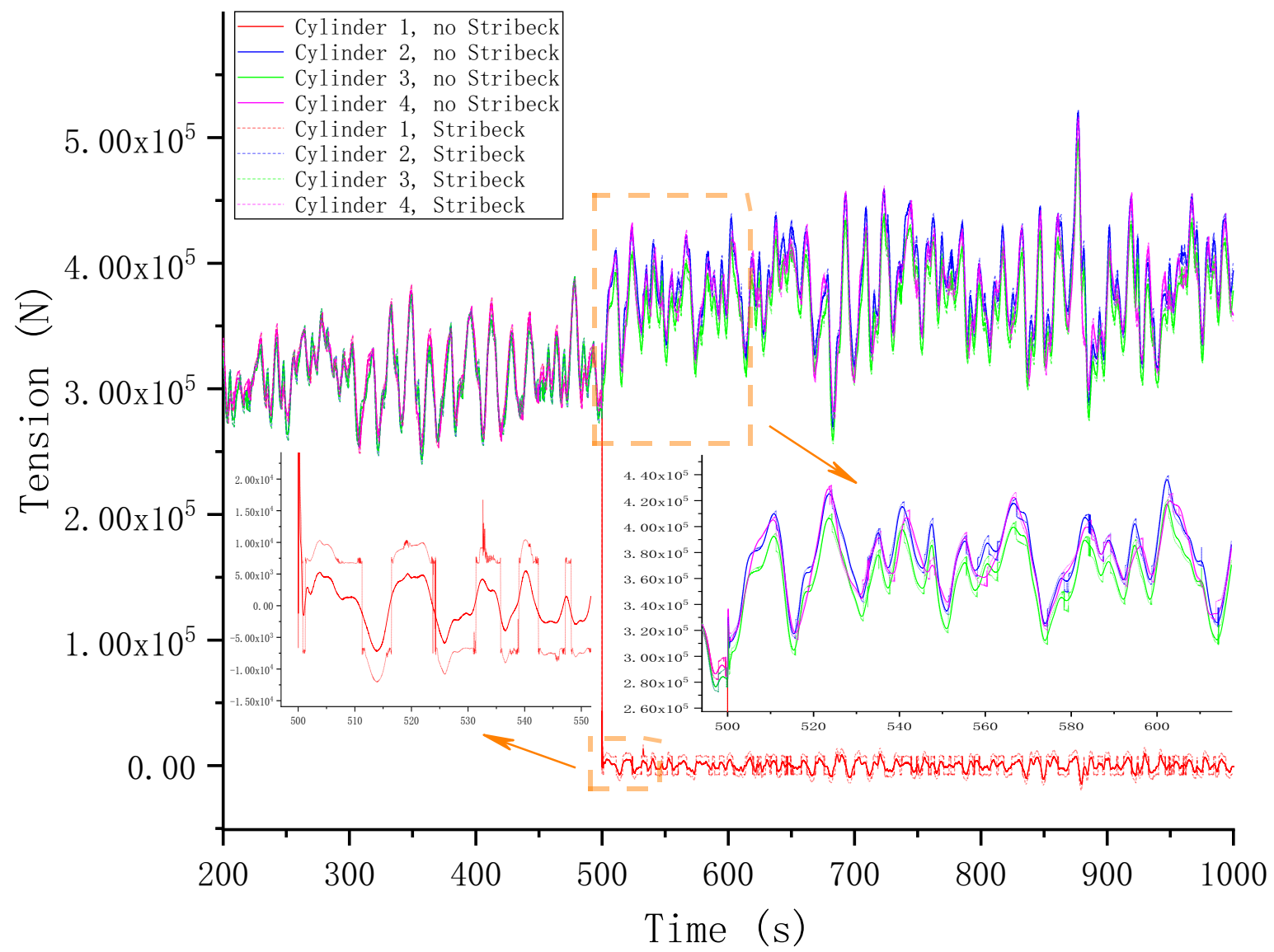

Figure 33 Time histories of tension on the cylinders, wave, current, and wind in $0^{\circ}$, under the failure of Cylinder 1 


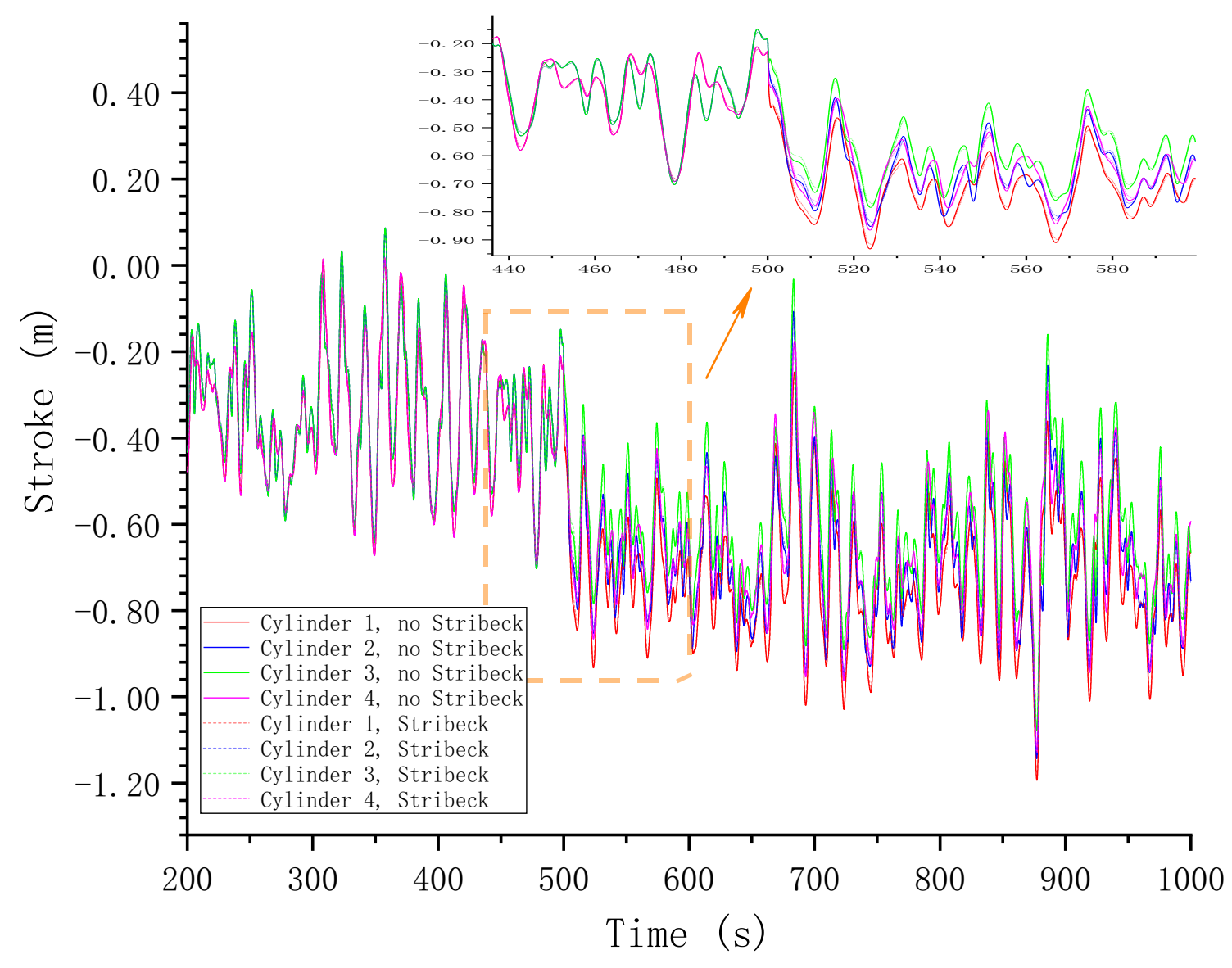

Figure 34 Time histories of strokes of the cylinders, wave, current, and wind in $0^{\circ}$, under the failure of Cylinder 1

\section{Wave, Current, and Wind in $45^{\circ}$}

Under the wave, current, and wind in $45^{\circ}$, the motion response of the hull and deflections of the risers after the tensioner failure (chosen at a representative instant as a hint) are shown in Figure 35, the time histories of the tensions acted on different cylinders of $R_{1}$ are shown in Figure 36 , and the time histories of their strokes are demonstrated in Figure 37. The tension and stroke responses with Stribeck model are demonstrated by the dashed lines in the figures. In the healthy stage, the tension of cylinders varies from $240.3 \mathrm{kN}$ to $389.6 \mathrm{kN}$, which is $93.5 \%$ to $151.6 \%$ of the cylinder's initial pretension. While in the later steady stage, the tension of cylinders sees an increase and it varies from $257.1 \mathrm{kN}$ to $522.0 \mathrm{kN}$, which is $100.0 \%$ to $203.1 \%$ of the initial pretension. Between the two stages, in the transient stage, there are only small tension peaks of the cylinders: for Cylinder 1 and 4 at $331.2 \mathrm{kN}$, and for Cylinder 3 at $319.8 \mathrm{kN}$. With regard to the strokes of the cylinders, in the healthy stage, they vary from - $0.707 \mathrm{~m}$ to $0.070 \mathrm{~m}$. In the steady stage, they drop down and range from $-1.184 \mathrm{~m}$ to $-0.038 \mathrm{~m}$. No obvious stroke peaks of the cylinders are observed in the figure, either. As a result, no matter which direction the environmental loads are applied in, no tension or stroke excess occurs, which is based on the maximum tension or the highest and lowest strokes of each cylinder, even though the platform is experiencing the extreme sea loads, e.g. a 1000-yr tropical cyclone, and the transient impacts are considered in the study. In terms of the design information of the HPT, the maximum tension of each cylinder is $1572 \mathrm{kN}$, which is corresponding to the lowest stroke of $-2.134 \mathrm{~m}$. The highest stroke is limited at $0.610 \mathrm{~m}$. The responses of the failed tensioner are actually still far from the tension and motion limits. It is important for the remaining cylinders of the HPT that no other cylinder will fail subsequently when the first cylinder or its hydraulic pressure mechanism fails accidentally, and 3 healthy cylinders can maintain the TTR structure surviving both in the transient and steady stages of a tensioner local failure. There is a big difference from the local mooring failure of the TLP, which tells it is possible for the mooring system to develop a progressive failure, even a global failure under the extreme sea state (Yu et al., 2019). 


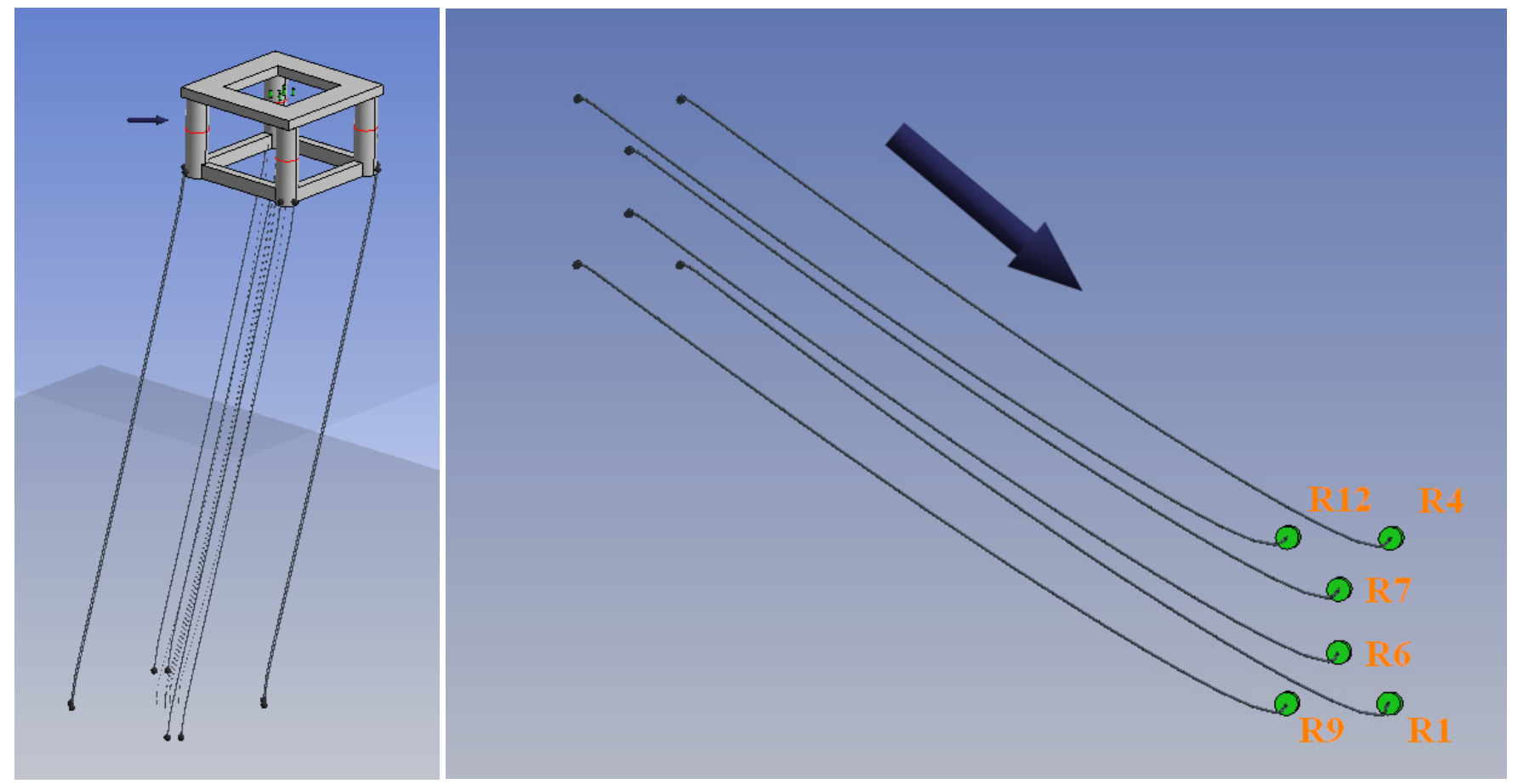

Figure 35 Motion response of the hull and axonometric projections of the risers, wave, current, and wind in $45^{\circ}$, under the failure of Cylinder 1 


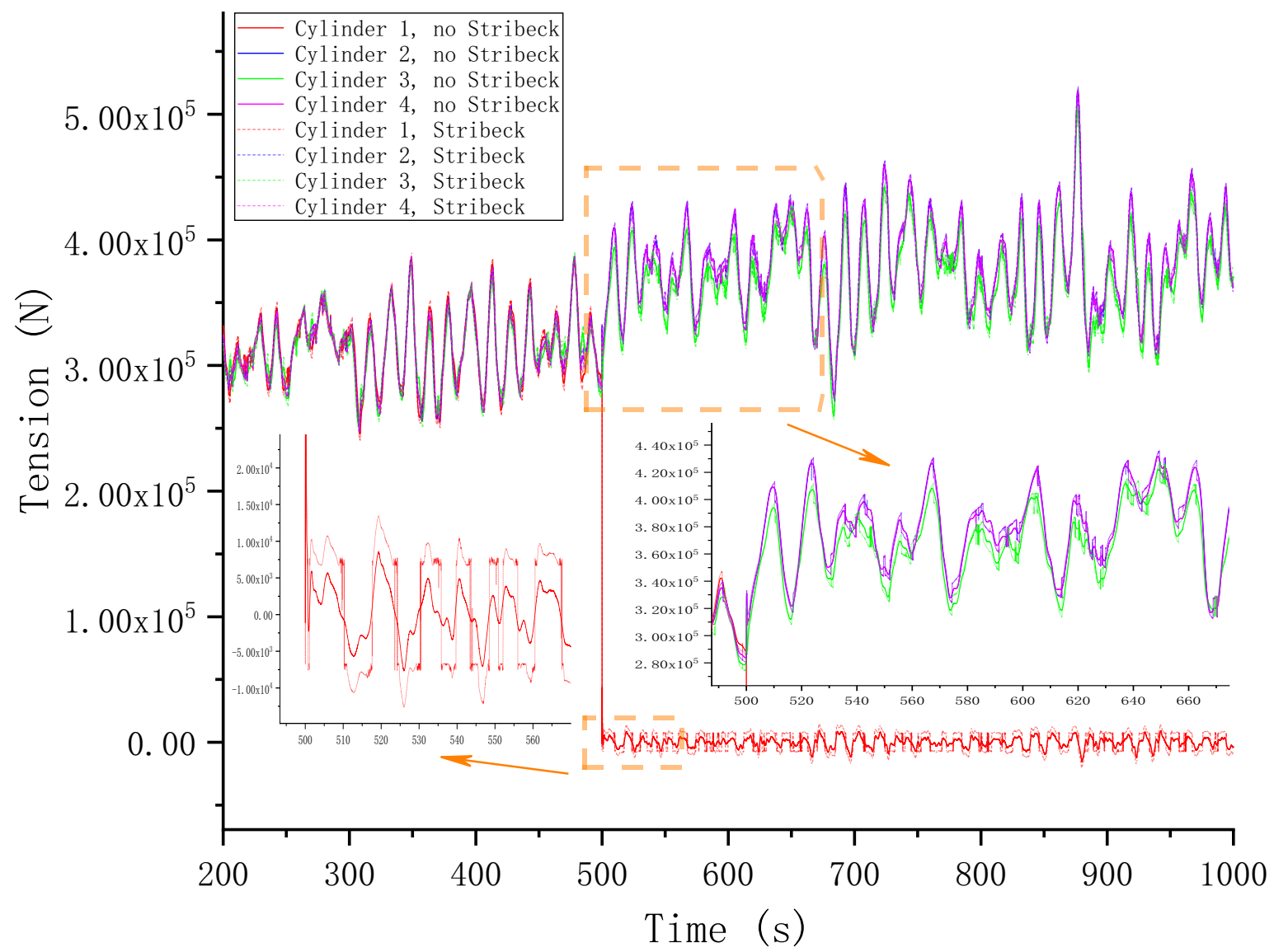

Figure 36 Time histories of tension on the cylinders, wave, current, and wind in $45^{\circ}$, under the failure of Cylinder 1 


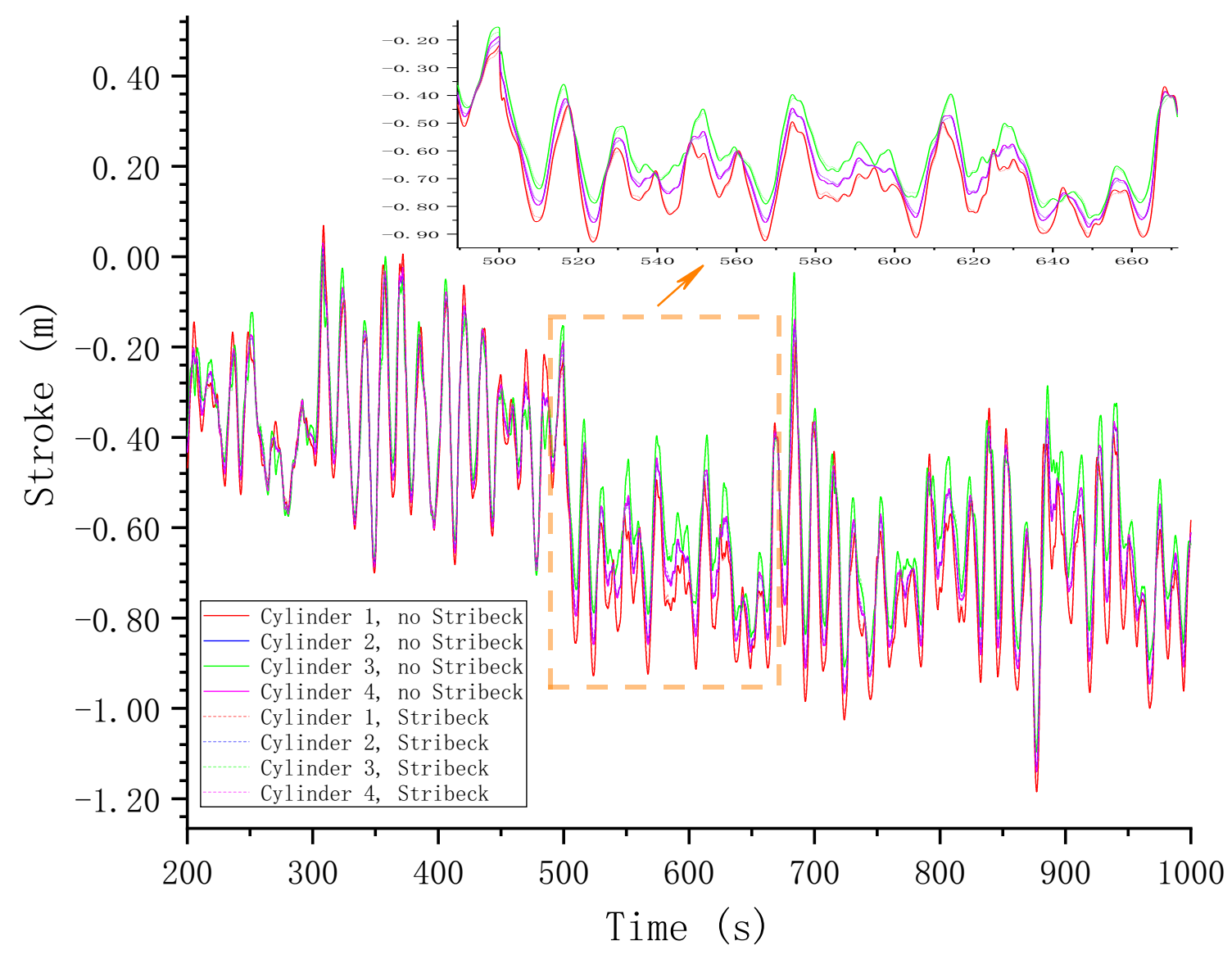

Figure 37 Time histories of strokes of the cylinders, wave, current, and wind in $45^{\circ}$, under the failure of Cylinder 1

\section{Summary and Conclusion}

The local failure of the HPT for risers is simulated in time domain by using a fully coupled TLP hull-tendon-TTR-tensioner system, which is proposed specially for solving the problem. An in-house developed FORTRAN subroutine is applied to simulate the multi-cylinder tensioner. A multi-TTR TLP model including 6 risers and tensioners is modeled to calculate the failed riser and other remaining risers simultaneously. The calm sea, the regular waves, and the extreme sea states with irregular waves, currents, and winds are all considered. The tensioner responses in different stages, including the healthy, transient, and steady stages, are analyzed and the difference between the responses is compared with and without the Stribeck friction. From the results of the simulations, the following conclusion can be drawn:

1) According to the different response features in the whole process of the HPT's local failure, it can be divided into 3 fundamental stages: the healthy stage, transient stage, and steady stage. The stabilized response of the healthy tensioner and riser is the starting point of their failure response. The transient stage is when the system experiences a transient impact on the tension or stroke due to the failure. It is quite short, and more intensive the external loads are, slighter the transient response is. In the steady stage, the HPT system finds out its new equilibrium point again. Finally, the failed TTR has a tilt at the top end.

2) In some situations of the HPT local failure, there is still a residual response of the tensioner and riser after the transient stage. The transient response can be propagated several cycles by the TTR between its top and bottom ends. When the propagated transient response goes back to the tensioner again, a residual response can be observed on the tensioner, even though the tensioner has entered the steady stage. 
3) There are transient interactions between different cylinders after a local failure occurs, which makes the transient stage more complicated. As for the transient response of each cylinder, they do not get to their final states under one stroke, and they do not response independently. As the HPT consists of 4 cylinders, one cylinder inevitably has an influence on the behaviors of other cylinders before they enter the steady stage. One dropping piston will drag other pistons to drop, and other pistons will also prevent it from dropping in return. Thus, there are some fluctuations in their transient responses.

4) The failure responses in the transient stage and the steady stage can be regarded as the approximate superposition of the response after the local failure in the calm sea and the healthy response under the environmental loads. In the healthy stage, the response shapes of the tension and strokes of the cylinders change significantly as the wave period changes, because the complicated coupled motion of the system is highly sensitive to the load period. While their response shapes in the steady stage are still almost same with those in the healthy stage.

5) It is hard for an accidental local failure of the HPT to develop into a progressive failure later. Even though the TLP is experiencing extreme environmental loads, the tension or strokes of the cylinders after the local failure occurs are still far from the limits of the HPT design, therefore, it cannot induce a second failure on other cylinders. Thus, enhancing the reliability of the hydraulic pneumatic system to avoid the initial accidental failure, instead of optimizing the structure and enhancing the structural redundancy of the HPT, is the key to reduce the loss due to the tensioner local failure.

In the future, the fully coupled TLP-TTR system modeled in the study also can be applied to the researches which focus on the TTR and HPT dynamic responses with consideration of the actual motion of the platform and the researches which focus on the design of active TTR tensioners. Widely, the proposed modeling and secondary development method can be transplanted to model other tautly moored floating structures with the actively adjustable mooring force, e.g. how to reduce the motion response of a TLP by active mooring control.

\section{Acknowledgements}

The authors would like to thank National Natural Science Foundation of China for Youth (Grant No. 51609169), GuangXi Science and Technology Major Project (Grant No. Guike AA17292007), National Key R\&D Program of China (Grant No. 2018YFC0310502), National Natural Science Foundation of China (Grant No. 51779173), and China Scholarship Council (CSC), for their financially support of the present research. They thank Mr. Yi Huang for language editing and proofreading this work.

\section{References}

Andersson, S., Söderberg, A., Björklund, S., 2007. Friction models for sliding dry, boundary and mixed lubricated contacts. Tribology international 40 (4), 580-587.

API, 2005. Recommended Practice for Planning, Design and Analysis of Stationkeeping Systems for Floating Structures (API-RP-2SK). American Petroleum Institute.

API, 2007. Recommended Practice for Planning Designing and Constructing Tension Leg Platforms (API-RP-2T). American Petroleum Institute.

Chen, B., Yu, J., Yu, Y., Xu, L., Hao, S., Wu, C., Wu, H., 2019. Study on key performance parameters of hydro-pneumatic tensioner for top tensioned riser. Applied Ocean Research 84, 206-215.

Chen, B., Yu, J., Yu, Y., Xu, L., Wu, H., Li, Z., 2018a. Modeling Approach of Hydropneumatic Tensioner for Top-Tensioned Riser. Journal of Offshore Mechanics and Arctic Engineering 140 (5), 051706.

Chen, B., Yu, Y., Yu, J., Xu, L., Hao, S., 2018b. Mathematical Model of Hydro-Pneumatic Tensioner for Top Tensioning Riser. Shipbuilding of China 59 (01), 151-160.

Cummins, W., 1962. The impulse response function and ship motions. David Taylor Model Basin Washington DC.

Faltinsen, O., 1993. Sea loads on ships and offshore structures. Cambridge university press.

Kim, C., Kim, M., Liu, Y., Zhao, C., 1994. Time domain simulation of nonlinear response of a coupled TLP system in random seas, The Fourth International Offshore and Polar Engineering Conference. International Society of Offshore and Polar Engineers.

Lee, C., Newman, J., 1991. The computation of second-order wave loads.

Lee, H., Roh, M.-I., Ham, S.-H., Ha, S., 2015. Dynamic simulation of the wireline riser tensioner system for a mobile offshore drilling unit based on multibody system dynamics. Ocean Engineering 106, 485-495.

Marton, L., Lantos, B., 2007. Modeling, identification, and compensation of stick-slip friction. IEEE Transactions on Industrial Electronics 54 (1), 511521.

Tang, Y., 2002. Advanced Structural Dynamics. Tianjin University Press, Tianjin, PRC.

Taylor, R.E., Jefferys, E., 1986. Variability of hydrodynamic load predictions for a tension leg platform. Ocean Engineering 13 (5), $449-490$.

Tran, X.B., Hafizah, N., Yanada, H., 2012. Modeling of dynamic friction behaviors of hydraulic cylinders. Mechatronics 22 (1), 65-75.

Wang, B., 2007. Basic Tutorial for Fortran 95 Program Design and Data Structure. Tianjin University Press, Tianjin, PRC.

Wang, T., Liu, Y., 2018. Dynamic response of platform-riser coupling system with hydro-pneumatic tensioner. Ocean Engineering 166, $172-181$.

Yanada, H., Sekikawa, Y., 2008. Modeling of dynamic behaviors of friction. Mechatronics 18 (7), 330-339. 
Yu, J., Hao, S., Yu, Y., Chen, B., Cheng, S., Wu, J., 2019. Mooring analysis for a whole TLP with TTRs under tendon one-time failure and progressive failure. Ocean Engineering 182, 360-385. 